\title{
ANALYSIS OF A SPECTRAL-GALERKIN APPROXIMATION TO THE HELMHOLTZ EQUATION IN EXTERIOR DOMAINS*
}

\author{
JIE SHEN $^{\dagger}$ AND LI-LIAN WANG ${ }^{\ddagger}$
}

\begin{abstract}
An error analysis is presented for the spectral-Galerkin method to the Helmholtz equation in 2- and 3-dimensional exterior domains. The problem in unbounded domains is first reduced to a problem on a bounded domain via the Dirichlet-to-Neumann operator, and then a spectral-Galerkin method is employed to approximate the reduced problem. The error analysis is based on exploring delicate asymptotic behaviors of the Hankel functions and on deriving a priori estimates with explicit dependence on the wave number for both the continuous and the discrete problems. Explicit error bounds with respect to the wave number are derived, and some illustrative numerical examples are also presented.
\end{abstract}

Key words. Helmholtz equation, wave scattering, error analysis, spectral-Galerkin, unbounded domain

AMS subject classifications. $65 \mathrm{~N} 35,65 \mathrm{~N} 22,65 \mathrm{~F} 05,35 \mathrm{~J} 05$

DOI. $10.1137 / 060665737$

1. Introduction. We consider in this paper the acoustic wave scattering from a bounded obstacle $D \subset \mathbb{R}^{d}, d=2,3$. In this case, the scattered wave satisfies the Helmholtz equation

$$
-\Delta U-k^{2} U=F \text { in } \mathbb{R}^{d} \backslash \bar{D},
$$

along with the Sommerfeld radiation condition at infinity

$$
\partial_{r} U-\mathrm{i} k U=o\left(r^{\frac{1-d}{2}}\right) \quad \text { as } r \rightarrow \infty, d=2,3,
$$

which ensures that waves do not reflect from the far field. On the surface of the scatterer $D$, a Dirichlet (sound soft) or Neumann (sound hard) condition is assumed.

Although the Helmholtz equation with (1.2) is linear, its numerical approximation and associated analysis are notoriously difficult due to the following: (i) the domain is unbounded; (ii) the system is not positive definite; and (iii) when the wave number $k \gg 1$, the solution is highly oscillatory. In particular, it remains a challenge to design numerical algorithms which are robust and efficient for moderate to high wave numbers.

There has been extensive research work devoted to overcoming these difficulties (see, for instance, $[16,23,22]$ and the references therein). In particular, it has been shown, at least for some simple cases, that errors of $p$ th order numerical methods for the Helmholtz equation behave like $O\left(k^{p+1} h^{p}\right)$ (see, for instance, $[18,4,30]$ ). Hence, high-order methods are particularly preferable for this type of problem over low-order methods. We note also that some very detailed analyses were carried out in $[2,3]$

${ }^{*}$ Received by the editors July 21, 2006; accepted for publication (in revised form) May 11, 2007; published electronically August 31, 2007.

http://www.siam.org/journals/sinum/45-5/66573.html

${ }^{\dagger}$ Department of Mathematics, Purdue University, West Lafayette, IN 47907 (shen@math.purdue. edu). The work of this author was partially supported by NFS grants DMS-0311915 and DMS0610646.

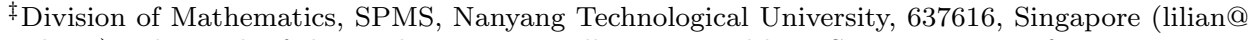
ntu.edu.sg). The work of this author was partially supported by a Start-Up grant of NTU.

1954 
on the discrete dispersive relation by the hp version of finite element method (FEM) and by the high-order discontinuous Galerkin method. These results indicate, once again, that high-order methods are preferable, if not necessary, for highly oscillatory problems.

On the other hand, the linear system from a discretization of the Helmholtz equation with moderate to high wave numbers is usually highly indefinite and difficult to solve. It is with these considerations in mind that we choose to use the transformed field expansion (TFE) method (cf. [26]), which improves over the classical field expansion method $[27,5,6]$, coupled with a fast spectral-Galerkin solution (cf. [28, 29, 24]).

There are a few recent works on wave number independent boundary element methods and on error estimates with explicit dependence on wave numbers for acoustic scattering problems. In [19, 8], the authors introduced a novel Galerkin boundary element method using a graded mesh and special basis functions and derived a quasi-optimal error estimate which is independent of wave number for the Helmholtz equation in a half-plane and exterior of a convex polygon.

We now briefly describe the TFE method for a 2-dimensional (2-D) obstacle enclosed by $\{r=a+g(\theta): 0 \leq \theta<2 \pi\}$. The TFE algorithm consists of the following steps:

- Assuming $F$ is compactly supported and choosing $b$ such that $b>a+$ $\max _{0 \leq \theta<2 \pi}|g(\theta)|$ and $\operatorname{supp} F \subset \Omega_{g}:=\{(r, \theta): a+g(\theta)<r<b\}$, we then use the Dirichlet-to-Neumann operator $T$ (see $[15,13]$ and the next section) to reduce the problem in the unbounded domain to

$$
\begin{array}{ll}
-\Delta U-k^{2} U=F & \text { in } \Omega_{g}, \\
\left.U\right|_{r=a+g(\theta)}=\xi, & \left.\left(\partial_{r} U+T(U)\right)\right|_{r=b}=0 .
\end{array}
$$

- Make a change of variables

$$
r^{\prime}=\frac{(b-a) r-b g(\theta)}{(b-a)-g(\theta)}, \quad \theta^{\prime}=\theta
$$

which maps $\Omega_{g}$ to an annulus $\Omega_{0}$. To simplify the notation, we still use $(r, \theta)$ to denote $\left(r^{\prime}, \theta^{\prime}\right)$ and $U, F, \xi$ to denote the functions $U, F, \xi$ after the change of variables. Then the problem (1.3) becomes

$$
\begin{aligned}
& -\Delta U-k^{2} U=F+J(g, U) \quad \text { in } \Omega_{0}, \\
& U(a, \theta)=\xi(\theta),\left.\quad\left(\partial_{r} U+T U\right)\right|_{r=b}=\eta(g, U),
\end{aligned}
$$

where $J(g, U)$ and $\eta(g, U)$ contain differential operators with nonconstant coefficients for which a fast direct/iterative solution is not available.

- Consider the obstacle $\{(r, \theta): r<a+g(\theta)\}$ as a perturbation of the disk $\{r<a\}$; i.e., write $g=\varepsilon h$ and expand $u$ as

$$
U(r, \theta ; \varepsilon)=\sum_{n=0}^{\infty} U_{n}(r, \theta) \varepsilon^{n}
$$

Plugging the above expansion into (1.5) and collecting terms with $\varepsilon^{n}$, we find that [24]

$$
\begin{aligned}
& -\Delta U_{n}-k^{2} U_{n}=\delta_{n, 0} F+\tilde{J}\left(g, U_{n-4}, \ldots, U_{n-1}\right) \quad \text { in } \Omega_{0}, \\
& U_{n}(a, \theta)=\delta_{n, 0} \xi(\theta),\left.\quad\left(\partial_{r} U_{n}+T U_{n}\right)\right|_{r=b}=\tilde{\eta}\left(g, U_{n-1}\right) .
\end{aligned}
$$

Copyright $@$ by SIAM. Unauthorized reproduction of this article is prohibited. 
- Solve (1.6) for $n=0,1,2, \ldots$, and sum up the series by using a Padé approximation.

It is shown in $[25,26]$ that this TFE method is stable and robust at high order, and it is demonstrated in [24] that this method, coupled with a spectral-Galerkin solution for (1.6), is very efficient and capable of providing very accurate results for bounded obstacle scattering with moderate to high wave numbers.

Notice that the whole algorithm boils down to solving a sequence of the following nonhomogeneous Helmholtz equation in an annulus (2-D) or a spherical shell (3-D):

$$
\begin{aligned}
& -\Delta U-k^{2} U=F \quad \text { in } \Omega_{0}, \\
& U(a, \theta)=\xi(\theta),\left.\quad\left(\partial_{r} U+T U\right)\right|_{r=b}=\eta(\theta) .
\end{aligned}
$$

The purpose of this paper is to present a detailed error analysis of the spectralGalerkin method for (1.7). The main difficulty here is to obtain error estimates with explicit dependence on the wave number. Among the very few results available in this regard are those in $[18,30]$, where the Helmholtz equation in bounded domains with a first-order approximation to the radiation boundary condition was considered and error estimates with explicit dependence on the wave numbers were derived. To the authors' best knowledge, there seems to be no rigorous error estimate available with explicit dependence on the wave number for a numerical scheme to bounded obstacle scattering.

We now introduce some notations to be used throughout this paper. Let $\varpi$ be a given positive weight function in $I:=(a, b)$. We denote by $L_{\varpi}^{2}(I)$ a Hilbert space of real or complex functions with inner product and norm

$$
(u, v)_{\varpi}=\int_{I} u(r) \overline{v(r)} \varpi(r) d r, \quad\|u\|_{\varpi}=\sqrt{(u, u)_{\varpi}},
$$

respectively, where $\bar{v}$ is the complex conjugate of $v$. Then the weighted Sobolev spaces $H_{\varpi}^{s}(I)(s=0,1,2, \ldots)$ can be defined as usual with inner products, norms, and seminorms denoted by $(\cdot, \cdot)_{s, \varpi},\|\cdot\|_{s, \varpi}$, and $|\cdot|_{s, \varpi}$, respectively. For real $s>0$, $H_{\varpi}^{s}(I)$ is defined by space interpolation (cf. [20]). The subscript $\varpi$ will be omitted from the notations in the case of $\varpi \equiv 1$. For simplicity, we denote $\partial_{r}^{l} v=\frac{d^{l} v}{d r^{l}}, l \geq 1$. We shall also use $(\cdot, \cdot)_{\omega}$ and $\|\cdot\|_{\omega}$ to denote the weighted inner product and the weighted $L^{2}$-norm, respectively, in two and three dimensions.

Let $S$ be the unit circle in 2-D and the unit sphere in 3-D; we also use the nonisotropic periodic-type Sobolev space on $\Omega=S \times I: H_{p}^{s^{\prime}}\left(S ; H_{\varpi}^{s}(I)\right), \quad s^{\prime} \geq 0$ (subscript $p$ stands for periodicity in the $\theta$-direction) with the norm

$$
\|U\|_{H_{p}^{s^{\prime}}\left(S ; H_{\varpi}^{s}(I)\right)}= \begin{cases}\left(\sum_{|m|=0}^{\infty}\left(1+m^{2}\right)^{s^{\prime}}\left\|\hat{u}_{m}\right\|_{s, \varpi}^{2}\right)^{1 / 2} & \text { if } d=2, \\ \left(\sum_{m=0}^{\infty} \sum_{l=-m}^{m}(1+m)^{2 s^{\prime}}\left\|\hat{u}_{l m}\right\|_{s, \varpi}^{2}\right)^{1 / 2} & \text { if } d=3,\end{cases}
$$

where $\left\{\hat{u}_{m}\right\}$ (resp., $\left\{\hat{u}_{l m}\right\}$ ) are the expansion coefficients of $U$ in terms of Fourier (resp., spherical harmonic) basis, i.e.,

$$
U=\sum_{|m|=0}^{\infty} \hat{u}_{m} e^{i m \theta} \quad \text { or } \quad U=\sum_{m=0}^{\infty} \sum_{l=-m}^{m} \hat{u}_{l m} Y_{m}^{l}(\theta, \phi) .
$$

The norm of the Sobolev space $H_{p}^{s^{\prime}}(S)$ on $S$ can be defined in the same fashion by replacing the norm $\|\cdot\|_{s, \varpi}$ by the absolute value $|\cdot|$. In particular, we have

$$
L_{p}^{2}(\Omega)=H_{p}^{0}\left(S, H_{\varpi}^{0}(I)\right) \text { with } \varpi=r^{d-1}, \quad d=2,3 .
$$


We assume that $a>0$ is a fixed parameter representing the radius of the scatterer. Throughout this paper, we denote by $c$ a generic positive constant which depends only on $a$ and possibly on a fixed $k_{0}>0$. We use the expression $A \lesssim B$ to mean that $A \leq c B$.

2. Dirichlet-to-Neumann (DtN) map. The error analysis relies heavily on the properties of the DtN map which we investigate below.

2.1. Formulation of the DtN operator. We start with the 3-D case and consider an "auxiliary" exterior problem

$$
\begin{cases}-\Delta U-k^{2} U=0 & \text { in } \Omega_{\mathrm{ext}}:=\mathbb{R}^{3} \backslash \bar{B} \\ U=\Psi & \text { on } \partial B\end{cases}
$$

where $B$ is a ball of radius $b$. This problem can be solved analytically via separation of variables; namely, we can express its solution as

$$
U(r, \theta, \phi)=\sum_{m=0}^{\infty} h_{m}^{(1)}(k r) \sum_{l=-m}^{m} \hat{u}_{l m} Y_{m}^{l}(\theta, \phi),
$$

where $(r, \theta, \phi) \in[b, \infty) \times[0,2 \pi) \times[0, \pi), h_{m}^{(1)}(z)$ is the spherical Hankel function of the first kind of order $m$, and $\left\{Y_{m}^{l}\right\}$ are the spherical harmonic functions. To determine the coefficients $\left\{\hat{u}_{l m}\right\}$, we expand the Dirichlet boundary value $\Psi$ on the sphere $\partial B$ as

$$
U(b, \theta, \phi)=\Psi(\theta, \phi)=\sum_{m=0}^{\infty} \sum_{l=-m}^{m} \hat{\psi}_{l m} Y_{m}^{l}(\theta, \phi)
$$

Letting $r=b$ in (2.2) and comparing the coefficients of the two expansions yield that

$$
\hat{u}_{l m}=\frac{\hat{\psi}_{l m}}{h_{m}^{(1)}(k b)} \quad \text { for } \quad m \geq|l| \geq 0 .
$$

Plugging it into (2.2) leads to the exact solution of (2.1):

$$
U(r, \theta, \phi)=\sum_{m=0}^{\infty} \frac{h_{m}^{(1)}(k r)}{h_{m}^{(1)}(k b)} \sum_{l=-m}^{m} \hat{\psi}_{l m} Y_{m}^{l}(\theta, \phi) .
$$

Differentiating (2.5) with respect to $r$ and setting $r=b$, we find

$$
\partial_{r} U(b, \theta, \phi)=\sum_{m=0}^{\infty} k \frac{h_{m}^{(1)^{\prime}}(k b)}{h_{m}^{(1)}(k b)} \sum_{l=-m}^{m} \hat{\psi}_{l m} Y_{m}^{l}(\theta, \phi) .
$$

Hence, the DtN map is defined explicitly as

$$
T(U)=\left.\frac{\partial U}{\partial \mathbf{n}}\right|_{\partial B}=-\left.\frac{\partial U}{\partial r}\right|_{r=b}=-\sum_{m=0}^{\infty} k \frac{h_{m}^{(1)^{\prime}}(k b)}{h_{m}^{(1)}(k b)} \sum_{l=-m}^{m} \hat{\psi}_{l m} Y_{m}^{l}(\theta, \phi),
$$

where $\mathbf{n}$ is the outward normal of $\Omega_{\text {ext }}$. 
The counterpart of $(2.1)$ in $2-\mathrm{D}$ is

$$
\begin{cases}-\Delta U-k^{2} U=0 & \text { in } \Omega_{\mathrm{ext}}:=\mathbb{R}^{2} \backslash \bar{B} \\ U=\Phi & \text { on } \partial B\end{cases}
$$

where $B$ is a circle of radius $b$ which can be solved analytically with the exact solution

$$
U(r, \theta)=\sum_{|m|=0}^{\infty} \hat{u}_{m} H_{m}^{(1)}(k r) e^{\mathrm{i} m \theta} \quad \forall(r, \theta) \in[b, \infty) \times[0,2 \pi) .
$$

Here $H_{m}^{(1)}(z)$ is the Hankel function of the first kind of order $m$. The coefficients $\left\{\hat{u}_{m}\right\}$ are determined by the boundary value $\Phi(\theta)$ with the expansion

$$
U(b, \theta)=\Phi(\theta)=\sum_{|m|=0}^{\infty} \hat{\phi}_{m} e^{\mathrm{i} m \theta} .
$$

Hence, letting $r=b$ in (2.9) and comparing the coefficients of the above two expansions lead to $\hat{u}_{m}=\hat{\phi}_{m} / H_{m}^{(1)}(k b)$. As a consequence, the exact solution of $(2.8)$ is

$$
U(r, \theta)=\sum_{|m|=0}^{\infty} \frac{H_{m}^{(1)}(k r)}{H_{m}^{(1)}(k b)} \hat{\phi}_{m} e^{\mathrm{i} m \theta} \quad \forall(r, \theta) \in[b, \infty) \times[0,2 \pi) .
$$

The 2-D DtN map is given by

$$
T(U)=\left.\frac{\partial U}{\partial \mathbf{n}}\right|_{\partial B}=-\left.\frac{\partial U}{\partial r}\right|_{r=b}=-\sum_{|m|=0}^{\infty} k \frac{H_{m}^{(1)^{\prime}}(k b)}{H_{m}^{(1)}(k b)} \hat{\phi}_{m} e^{\mathrm{i} m \theta} .
$$

By using the DtN map $T$ and choosing $b$ sufficiently large so that $B$ contains both $D$ and $\operatorname{supp} F$, the original problem (1.1)-(1.2) with a Dirichlet boundary condition is reduced to:

$$
\begin{cases}-\Delta U-k^{2} U=F & \text { in } \Omega:=B \cap \mathbb{R}^{d} \backslash \bar{D}, d=2,3, \\ U=\xi & \text { on } \partial D, \\ \partial_{r} U+T U=0 & \text { on } \partial B .\end{cases}
$$

To fix the idea, we prescribed a Dirichlet boundary condition on the scatterer $D$; other types of boundary conditions can be used as well.

2.2. Properties of the DtN kernel. In order to carry out a rigorous mathematical analysis for the problem (2.13), we need to study carefully the properties of the DtN kernel associated with (2.7) and (2.12), i.e., the properties of the coefficients:

$$
\mathcal{T}_{m, \kappa}= \begin{cases}\frac{H_{m}^{(1)^{\prime}}(\kappa)}{{H_{m}^{(1)}}^{(1)}(\kappa)} & \text { if } d=2, \\ \frac{h_{m}^{(1)^{\prime}}(\kappa)}{h_{m}^{(1)}(\kappa)} & \text { if } d=3 .\end{cases}
$$

Copyright $@$ by SIAM. Unauthorized reproduction of this article is prohibited. 
2.2.1. Behavior of the $3-\mathbf{D}$ kernel. In this case, we have $\kappa>0$ and $m \geq 0$. We recall that

$$
h_{m}^{(1)}(\kappa)=j_{m}(\kappa)+\mathrm{i} y_{m}(\kappa)=\sqrt{\frac{\pi}{2 \kappa}} J_{m+1 / 2}(\kappa)+\mathrm{i} \sqrt{\frac{\pi}{2 \kappa}} Y_{m+1 / 2}(\kappa),
$$

where $J_{\nu}$ and $Y_{\nu}$ (resp., $j_{\nu}$ and $y_{\nu}$ ) are the Bessel (resp., spherical Bessel) functions of the first and second kinds, respectively, of order $\nu$. Using the relevant properties of the Bessel functions (cf. [31]), one verifies that

$$
\begin{aligned}
\operatorname{Re}\left(\mathcal{T}_{m, \kappa}\right) & =\frac{m}{\kappa}-\frac{j_{m}(\kappa) j_{m+1}(\kappa)+y_{m}(\kappa) y_{m+1}(\kappa)}{\left|h_{m}^{(1)}(\kappa)\right|^{2}} \\
& =\frac{m}{\kappa}-\frac{J_{m+1 / 2}(\kappa) J_{m+3 / 2}(\kappa)+Y_{m+1 / 2}(\kappa) Y_{m+3 / 2}(\kappa)}{J_{m+1 / 2}^{2}(\kappa)+Y_{m+1 / 2}^{2}(\kappa)} ; \\
\operatorname{Im}\left(\mathcal{T}_{m, \kappa}\right) & =\frac{1}{\kappa^{2}\left|h_{m}^{(1)}(\kappa)\right|^{2}}=\frac{2}{\pi \kappa} \frac{1}{J_{m+1 / 2}^{2}(\kappa)+Y_{m+1 / 2}^{2}(\kappa)} .
\end{aligned}
$$

An explicit expression of $\mathcal{T}_{m, \kappa}$ is given by Theorem 2.6.1 of [23]:

$$
\mathcal{T}_{m, \kappa}=\operatorname{Re}\left(\mathcal{T}_{m, \kappa}\right)+\mathrm{i} \operatorname{Im}\left(\mathcal{T}_{m, \kappa}\right)=-\frac{P_{m}(\kappa)}{\kappa Q_{m}(\kappa)}+\frac{\mathrm{i}}{Q_{m}(\kappa)},
$$

where

$$
\begin{aligned}
& P_{m}(\kappa)=1+2 a_{1}^{m} \frac{1}{\kappa^{2}}+\cdots+(m+1) a_{m}^{m} \frac{1}{\kappa^{2 m}} \\
& Q_{m}(\kappa)=1+a_{1}^{m} \frac{1}{\kappa^{2}}+\cdots+a_{m}^{m} \frac{1}{\kappa^{2 m}},
\end{aligned}
$$

with

$$
a_{j}^{m}=\frac{(m+j) !(2 j) !}{4^{j}(j !)^{2}(m-j) !} .
$$

We now study the monotonic property of $\operatorname{Im}\left(\mathcal{T}_{m, \kappa}\right)$ with respect to $m$ and $\kappa$. We observe from (2.17)-(2.19) that, for a fixed $m \geq 0, \operatorname{Im}\left(\mathcal{T}_{m, \kappa}\right)$ is an increasing function of $\kappa$, as illustrated by Figure 2.1(b). However, for a given $\kappa>0, \operatorname{Im}\left(\mathcal{T}_{m, \kappa}\right)$ is a decreasing function of $m$, which follows from Nicholson's formula (see p. 444 of [31])

$$
J_{m+1 / 2}^{2}(\kappa)+Y_{m+1 / 2}^{2}(\kappa)=\frac{8}{\pi^{2}} \int_{0}^{+\infty} \mathcal{K}_{0}(2 \kappa \sinh t) \cosh ((2 m+1) t) d t,
$$

where $\mathcal{K}_{0}(\xi)>0$ is Kelvin's function defined by (A.2) in the appendix.

We next consider the bounds and asymptotic behavior of $\mathcal{T}_{m, \kappa}$. An immediate consequence of (2.17)-(2.19) is that

$$
\operatorname{Re}\left(\mathcal{T}_{m, \kappa}\right)<0, \quad \operatorname{Im}\left(\mathcal{T}_{m, \kappa}\right)>0,
$$

which ensures the well-posedness of the problem (2.13) (cf. [11]). Moreover, we have the following bounds (see, e.g., p. 87 of [23]):

$$
-\frac{m+1}{\kappa} \leq \operatorname{Re}\left(\mathcal{T}_{m, \kappa}\right) \leq-\frac{1}{\kappa}, \quad 0<\operatorname{Im}\left(\mathcal{T}_{m, \kappa}\right) \leq 1,
$$

Copyright (c) by SIAM. Unauthorized reproduction of this article is prohibited. 

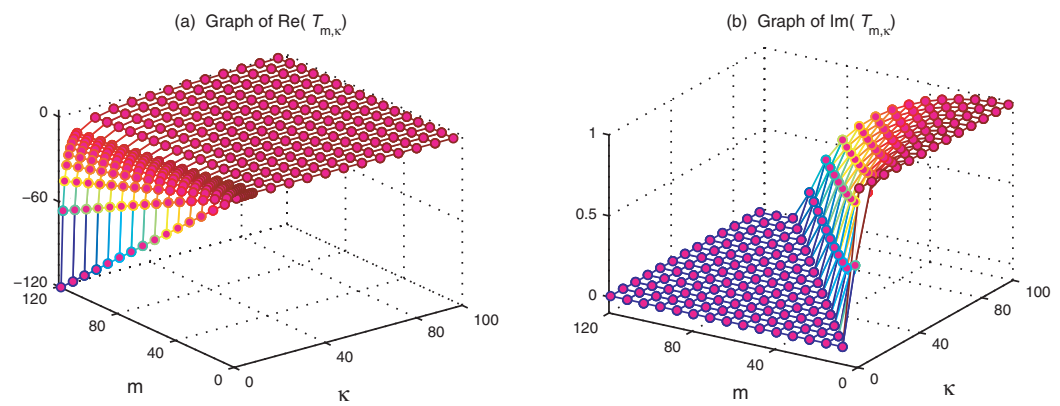

FIG. 2.1. Graphs of $\operatorname{Re}\left(\mathcal{T}_{m, \kappa}\right)$ and $\operatorname{Im}\left(\mathcal{T}_{m, \kappa}\right)$, with $(\kappa, m) \in[1,100] \times[0,120]$, in the $3-D$ case.
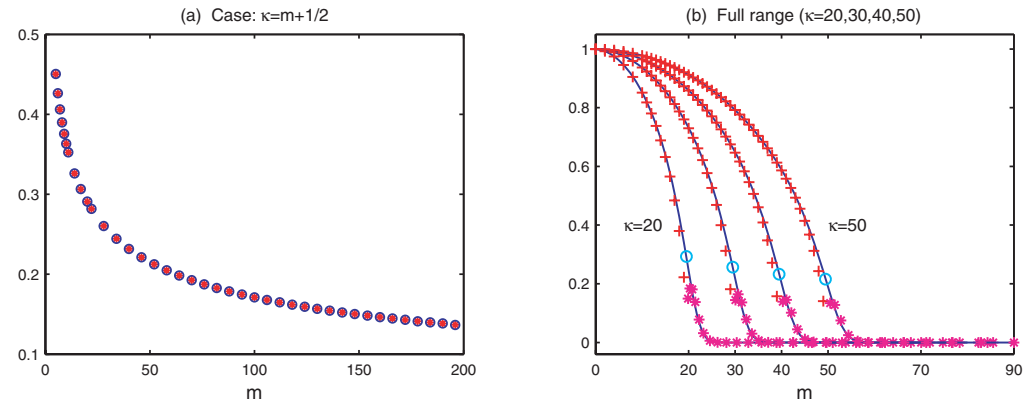

FIG. 2.2. (a) $\operatorname{Im}\left(\mathcal{T}_{m, m+1 / 2}\right)(\star)$ against $E_{m, m+1 / 2}(\mathrm{O})$ with $m \in[1,200] ;$ (b) $\operatorname{Im}\left(\mathcal{T}_{m, \kappa}\right)$ (solid line) against $E_{m, \kappa}(+$ for $\kappa>m+1 / 2$, (O) for $k=m+1 / 2$, and $(\star)$ for $\kappa<m+1 / 2)$, with $\kappa=20,30,40,50$.

in particular, by (2.17)-(2.19),

$$
\operatorname{Re}\left(\mathcal{T}_{0, \kappa}\right)=-\frac{1}{\kappa}, \quad \operatorname{Im}\left(\mathcal{T}_{0, \kappa}\right)=1 .
$$

We now seek more precise estimates of $\operatorname{Im}\left(\mathcal{T}_{m, \kappa}\right)$ and proceed separately with three cases:

(i) $\kappa>m+1 / 2$. We first recall the estimate (see p. 447 of [31])

$$
\frac{2}{\pi \kappa}<J_{\nu}^{2}(\kappa)+Y_{\nu}^{2}(\kappa)<\frac{2}{\pi \sqrt{\kappa^{2}-\nu^{2}}} \quad \text { if } \quad \frac{1}{2} \leq \nu<\kappa,
$$

which, together with (2.16b), implies that

$$
E_{m, \kappa}:=\frac{\sqrt{\kappa^{2}-(m+1 / 2)^{2}}}{\kappa}<\operatorname{Im}\left(\mathcal{T}_{m, \kappa}\right)<1 \quad \text { if } \quad \kappa>m+\frac{1}{2} .
$$

We observe from Figure 2.2 that the lower bound $E_{m, \kappa}$ provides an acceptable approximation to $\operatorname{Im}\left(\mathcal{T}_{m, \kappa}\right)$.

(ii) $\quad \kappa=m+1 / 2$. Using the formulas (see p. 232 of [31])

$$
J_{\nu}(\nu)=C_{1} \nu^{-1 / 3}+O\left(\nu^{-5 / 3}\right), \quad Y_{\nu}(\nu)=-C_{2} \nu^{-1 / 3}+O\left(\nu^{-5 / 3}\right),
$$

with

$$
C_{1}=\frac{\Gamma(1 / 3)}{2^{2 / 3} 3^{1 / 6} \pi} \approx 0.4473, \quad C_{2}=\frac{3^{1 / 3} \Gamma(1 / 3)}{2^{2 / 3} \pi} \approx 0.7748,
$$

Copyright (c) by SIAM. Unauthorized reproduction of this article is prohibited. 
we obtain from (2.16b) that

$$
\operatorname{Im}\left(\mathcal{T}_{m, m+1 / 2}\right) \sim C_{\circ}(m+1 / 2)^{-1 / 3}:=E_{m, m+1 / 2},
$$

with $C_{\circ}=2 /\left(\pi\left(C_{1}^{2}+C_{2}^{2}\right)\right) \approx 0.7954$.

In Figure 2.2(a), we plot $\operatorname{Im}\left(\mathcal{T}_{m, m+1 / 2}\right)$ against $E_{m, m+1 / 2}$ for $1 \leq m \leq 200$, which shows that, even for small $m$, the asymptotic estimate $C_{\circ}(m+1 / 2)^{-1 / 3}$ provides a very good approximation to $\operatorname{Im}\left(\mathcal{T}_{m, m+1 / 2}\right)$.

(iii) $\quad \kappa<m+1 / 2$. By the asymptotic formulas (see p. 243 of [31])

$$
J_{\nu}(\nu \operatorname{sech} \alpha) \sim \frac{e^{\nu(\tanh \alpha-\alpha)}}{\sqrt{2 \pi \nu \tanh \alpha}}, \quad Y_{\nu}(\nu \operatorname{sech} \alpha) \sim-\frac{e^{\nu(\alpha-\tanh \alpha)}}{\sqrt{\frac{1}{2} \pi \nu \tanh \alpha}},
$$

one verifies that for $m+1 / 2=\kappa \cosh \alpha$, with $\alpha>0$,

$$
\operatorname{Im}\left(\mathcal{T}_{m, \kappa}\right) \sim \frac{2(2 m+1) \tanh \alpha}{\kappa\left[e^{(2 m+1)(\tanh \alpha-\alpha)}+4 e^{(2 m+1)(\alpha-\tanh \alpha)}\right]}:=E_{m, \kappa} .
$$

Hence, $\operatorname{Im}\left(\mathcal{T}_{m, \kappa}\right)$ becomes exponentially small for large $m$. The exponential decay of $\operatorname{Im}\left(\mathcal{T}_{m, \kappa}\right)$ is shown more clearly from the asymptotic estimate

$$
\operatorname{Im}\left(\mathcal{T}_{m, \kappa}\right) \sim\left(\frac{e \kappa}{2 m+1}\right)^{2 m}, \quad m \gg \kappa
$$

which follows from formula 9.3.1 of [1]:

$$
J_{\nu}(\kappa) \sim \frac{1}{\sqrt{2 \pi \nu}}\left(\frac{e \kappa}{2 \nu}\right)^{\nu}, \quad Y_{\nu}(\kappa) \sim-\frac{2}{\sqrt{\pi \nu}}\left(\frac{e \kappa}{2 \nu}\right)^{-\nu}, \nu \gg \kappa .
$$

We plot in Figure 2.2(b) the estimate $E_{m, \kappa}$ (defined in (2.25), (2.27), and (2.29)) versus $\operatorname{Im}\left(\mathcal{T}_{m, \kappa}\right)$, with $\kappa=20,30,40,50$ and various $m$, which indicates that $E_{m, \kappa}$ provides an accurate picture of $\operatorname{Im}\left(\mathcal{T}_{m, \kappa}\right)$.

2.2.2. Behavior of the 2-D kernel. The identity $H_{-\nu}^{(1)}(z)=e^{\nu \pi \mathrm{i}} H_{\nu}^{(1)}(z)$ and the definition (2.14) imply that

$$
\mathcal{T}_{-m, \kappa}=\frac{H_{-m}^{(1)^{\prime}}(\kappa)}{H_{-m}^{(1)}(\kappa)}=\frac{(-1)^{m} H_{m}^{(1)^{\prime}}(\kappa)}{(-1)^{m} H_{m}^{(1)}(\kappa)}=\mathcal{T}_{m, \kappa} .
$$

Hence, it suffices to consider $\mathcal{T}_{m, \kappa}$ with $m \geq 0$. Using the recursion formulas of the Bessel functions, one verifies that

$$
\begin{gathered}
\operatorname{Re}\left(\mathcal{T}_{m, \kappa}\right)=\frac{m}{\kappa}-\frac{J_{m}(\kappa) J_{m+1}(\kappa)+Y_{m}(\kappa) Y_{m+1}(\kappa)}{J_{m}^{2}(\kappa)+Y_{m}^{2}(\kappa)} ; \\
\operatorname{Im}\left(\mathcal{T}_{m, \kappa}\right)=\frac{2}{\pi \kappa} \frac{1}{\left|H_{m}^{(1)}(\kappa)\right|^{2}}=\frac{2}{\pi \kappa} \frac{1}{J_{m}^{2}(\kappa)+Y_{m}^{2}(\kappa)} .
\end{gathered}
$$

We observe that the 2-D kernel has an expression similar to that of the 3-D kernel (cf. (2.16)). In fact, they share similar properties and asymptotic behaviors except for $m=0$ (comparison: Figure 2.1(a) versus Figure 2.3(a) and Figure 2.2(b) versus Figure 2.3(b)).

Indeed, we notice that the same monotonic property holds for the $2-\mathrm{D} \operatorname{Im}\left(\mathcal{T}_{m, \kappa}\right)$ : 

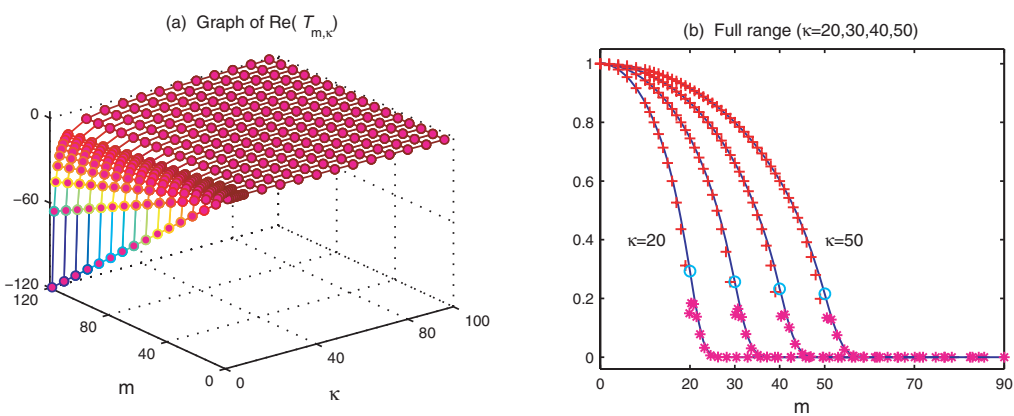

Fig. 2.3. (a) Graph of $2-D \operatorname{Re}\left(\mathcal{T}_{m, \kappa}\right)$, with $(\kappa, m) \in[1,100] \times[1,120]$; (b) $2-D \operatorname{Im}\left(\mathcal{T}_{m, \kappa}\right)$ (solid line) against $E_{m, \kappa}$ defined in (2.35) ( + for $\kappa>m+1 / 2$, ○ for $\kappa=m+1 / 2$, and $(\star)$ for $\left.\kappa<m+1 / 2\right)$, with $\kappa=20,30,40,50$.

(i) For a given $m \geq 1, \operatorname{Im}\left(\mathcal{T}_{m, \kappa}\right)$ is a strictly increasing function of $\kappa$, which follows from (2.33b) and the fact that $\kappa\left|H_{m}^{(1)}(\kappa)\right|^{2}$ is a strictly decreasing function of $\kappa$ (cf. p. 446 of [31]);

(ii) for a fixed $\kappa>0, \operatorname{Im}\left(\mathcal{T}_{m, \kappa}\right)$ is a strictly decreasing function of $m$, which is a direct consequence of Nicholson's formula (A.3a).

As in (2.22), we have the following bound for the 2-D kernel (see the appendix for the proof):

$$
\begin{gathered}
0<\operatorname{Im}\left(\mathcal{T}_{m, \kappa}\right)<1, m \geq 1 ; \\
-\frac{m}{\kappa} \leq \operatorname{Re}\left(\mathcal{T}_{m, \kappa}\right) \leq-\frac{1}{2 \kappa}, \quad m \geq 1 ; \quad-\frac{1}{2 \kappa} \leq \operatorname{Re}\left(\mathcal{T}_{0, \kappa}\right)<0 ; \\
\operatorname{Im}\left(\mathcal{T}_{0, \kappa}\right)>1 \quad \forall \kappa>0 .
\end{gathered}
$$

As in the 3-D case, applying the general formulas (2.24), (2.26), and (2.28) to the 2 -D $\operatorname{Im}\left(\mathcal{T}_{m, \kappa}\right)$, we find that an accurate approximation for $\operatorname{Im}\left(\mathcal{T}_{m, \kappa}\right)$ is

$$
E_{m, \kappa}:= \begin{cases}\sqrt{1-m^{2} / \kappa^{2}} & \text { if } \kappa>m \geq 1, \\ C_{\circ} m^{-1 / 3} & \text { if } \kappa=m, \\ \frac{1}{\kappa\left[e^{2 m(\tanh \alpha-\alpha)}+4 e^{2 m(\alpha-\tanh \alpha)}\right]} & \text { if } \kappa=m \operatorname{sech} \alpha, \alpha>0,\end{cases}
$$

where the constant $C_{\circ}$ is defined by $(2.27)$.

In Figure 2.3(b), we plot $E_{m, \kappa}$ against $\operatorname{Im}\left(\mathcal{T}_{m, \kappa}\right)$, which indicates that the estimate $E_{m, \kappa}$ gives an accurate picture of the behavior of $\operatorname{Im}\left(\mathcal{T}_{m, \kappa}\right)$.

3. A priori estimates. In order to carry out error analysis for the spectralGalerkin approximation to (1.7), we need to establish some a priori estimates for the solution of (1.7). Without loss of generality, we shall set $\xi=0$ since the nonhomogeneous boundary condition at $r=a$ can be simply converted to a homogeneous one by subtracting a suitable function from the solution.

3.1. Dimension reduction. We now rewrite (1.7) with $\xi=0$ in polar coordinates $(r, \theta)$ or spherical coordinates $(r, \theta, \phi)$ :

$$
\left\{\begin{array}{l}
-\left(\frac{1}{r^{d-1}} \partial_{r}\left(r^{d-1} \partial_{r} U\right)+\frac{1}{r^{2}} \Delta_{S} U\right)-k^{2} U=F \quad \text { in } \Omega=(a, b) \times S, \\
\left.U\right|_{r=a}=0,\left.\quad\left[\partial_{r} U+T(U)\right]\right|_{r=b}=\eta,
\end{array}\right.
$$

Copyright (c) by SIAM. Unauthorized reproduction of this article is prohibited. 
where

$$
\Delta_{S} U= \begin{cases}\partial_{\theta}^{2} U & \text { if } d=2, \\ \frac{1}{\sin ^{2} \phi} \partial_{\theta}^{2} U+\frac{1}{\sin \phi} \partial_{\phi}\left(\sin \phi \partial_{\phi} U\right) & \text { if } d=3,\end{cases}
$$

and its eigenfunctions are the Fourier basis $\left\{e^{\mathrm{i} m \theta}\right\}$ (in 2-D) or the spherical harmonic functions $\left\{Y_{m}^{l}(\theta, \phi)\right\}$ (in 3-D), i.e.,

$$
-\Delta_{S} e^{\mathrm{i} m \theta}=m^{2} e^{\mathrm{i} m \theta}(d=2) ; \quad-\Delta_{S} Y_{m}^{l}(\theta, \phi)=m(m+1) Y_{m}^{l}(\theta, \phi)(d=3) .
$$

We shall denote

$$
\beta_{m}=\left\{\begin{array}{lll}
m^{2}, & m=0, \pm 1, \pm 2, \ldots, & \text { if } d=2, \\
m(m+1), & m=0,1,2, \ldots, & \text { if } d=3 .
\end{array}\right.
$$

Expanding the solution and given data in terms of the eigenfunctions of $\Delta_{S}$ :

$$
(U, F, \eta)= \begin{cases}\sum_{|m|=0}^{\infty}\left(\hat{u}_{m}(r), \hat{f}_{m}(r), \hat{h}_{m}\right) e^{\mathrm{i} m \theta} & \text { if } d=2, \\ \sum_{m=0}^{\infty} \sum_{l=-m}^{m}\left(\hat{u}_{l m}(r), \hat{f}_{l m}(r), \hat{h}_{l m}\right) Y_{m}^{l}(\theta, \phi) & \text { if } d=3,\end{cases}
$$

we find from (3.3) that the problem (3.1)-(3.2) is reduced to the following sequence of 1-dimensional equations (for brevity, we use $u$ to denote $\hat{u}_{m}$ or $\hat{u}_{l m}$ and likewise for $f$ and $h$ below):

$$
\left\{\begin{array}{l}
-\frac{1}{r^{d-1}} \frac{d}{d r}\left[r^{d-1} \frac{d u}{d r}\right]+\beta_{m} \frac{u}{r^{2}}-k^{2} u=f, \quad r \in(a, b), d=2,3, \\
u(a)=0, \quad u^{\prime}(b)-k T_{m, k} u(b)=h,
\end{array}\right.
$$

where $T_{m, k}$ is derived from (2.7) and (2.12):

$$
T_{m, k}= \begin{cases}\frac{H_{m}^{(1)^{\prime}}(k b)}{H_{m}^{(1)}(k b)} & \text { if } d=2, \\ \frac{h_{m}^{(1)^{\prime}}(k b)}{h_{m}^{(1)}(k b)} & \text { if } d=3 .\end{cases}
$$

Notice that $T_{m, k}=\mathcal{T}_{m, k b}$ (defined by $(2.14)$ ).

3.2. Variational formulation and a priori estimates. We denote the weight functions $\omega^{\alpha}(r)=r^{\alpha}$ and $\omega(r)=r$. Define the 1-D weighted space

$$
X:=X(d)=\left\{u \in H_{\omega^{d-1}}^{1}(I) \cap L_{\omega^{d-3}}^{2}(I): u(a)=0\right\} .
$$

We define a bilinear form on $H_{p}^{1}(S ; X) \times H_{p}^{1}(S ; X)$ :

$$
\begin{aligned}
\mathcal{B}(U, V)= & \left(\partial_{r} U, \partial_{r} V\right)_{\omega^{d-1}}+\left(\nabla_{S} U, \nabla_{S} V\right)_{\omega^{d-3}}-k^{2}(U, V)_{\omega^{d-1}} \\
& +b^{d-1}\langle T(U)(\cdot), V(b, \cdot)\rangle_{S},
\end{aligned}
$$

where $\langle\cdot, \cdot\rangle_{S}$ is the $L^{2}(S)$-inner product (cf. the appendix), and the gradient operator $\nabla_{S}$ is defined by

$$
\nabla_{S} U= \begin{cases}\partial_{\theta} U & \text { if } d=2 \\ \left(\frac{1}{\sin \theta} \partial_{\theta} U\right) \vec{e}_{\theta}+\left(\partial_{\phi} U\right) \vec{e}_{\phi} & \text { if } d=3\end{cases}
$$

Copyright (C) by SIAM. Unauthorized reproduction of this article is prohibited. 
The variational formulation of (3.1) is as follows: Given $F \in L_{\omega^{d-1}}^{2}(\Omega)$ and $\eta \in$ $L^{2}(S)$, find $U \in H_{p}^{1}(S ; X)$ such that

$$
\mathcal{B}(U, V)=(F, V)_{\omega^{d-1}}+b^{d-1}\langle\eta, V(b, \cdot)\rangle_{S} \quad \forall V \in H_{p}^{1}(S ; X), \quad d=2,3,
$$

which admits a unique solution (see, e.g., [23]). The first main result of this paper is the following a priori estimates.

TheOrem 3.1. Let $U$ be the solution of (3.11). If $F \in L^{2}(\Omega)$ and $\eta \in L^{2}(S)$, then we have

$$
\|\nabla U\|+k\|U\|_{\Omega} \lesssim\left(\sqrt{b^{d}}+\sqrt{b|I|}(k b)^{1 / 3}\right)\|\eta\|_{L^{2}(S)}+(k b)^{1 / 3}|I|\|F\|,
$$

where $|I|=b-a$.

The rest of this section is devoted to the proof of this estimate. Observe that, for each mode $m$ or $(l, m)$, the expansion coefficient $u=\hat{u}_{m}$ or $\hat{u}_{l m}$ (cf. (3.5)) satisfies the following reduced problem (i.e., the variational formulation of (3.6)-(3.7)):

$$
\begin{aligned}
& \text { Given } f \in L_{\omega^{d-1}}^{2}(I) \text { and } h \in \mathbb{C} \text {, find } u \in X \text { such that } \\
& \mathcal{B}_{m}(u, v)=(f, v)_{\omega^{d-1}}+b^{d-1} h \overline{v(b)} \quad \forall v \in X, \quad d=2,3,
\end{aligned}
$$

where $f=\hat{f}_{m}$ or $\hat{f}_{l m}, h=\hat{h}_{m}$ or $\hat{h}_{l m}$, and the sesquilinear form

$$
\mathcal{B}_{m}(u, v):=\left(\partial_{r} u, \partial_{r} v\right)_{\omega^{d-1}}+\beta_{m}(u, v)_{\omega^{d-3}}-k^{2}(u, v)_{\omega^{d-1}}-k b^{d-1} T_{m, k} u(b) \overline{v(b)},
$$

where $\beta_{m}$ is defined in (3.4).

An essential step is to derive a priori estimates for each $u=\hat{u}_{m}$ or $\hat{u}_{l m}$ and then combine these estimates to get the desired result for the original problem (3.11).

We have the following a priori estimate for the solution of (3.13)-(3.14).

LEMma 3.1. Let $|I|=b-a$ be the length of the interval $I=(a, b)$. If $f \in L_{\omega^{d-1}}^{2}(I)$, then given $k_{0}>0$, we have that, for $k \geq k_{0}$ and $d=2,3$,

$$
\begin{aligned}
\left\|\partial_{r} u\right\|_{\omega^{d-1}} & +\sqrt{\beta_{m}}\|u\|_{\omega^{d-3}}+k\|u\|_{\omega^{d-1}} \\
& \lesssim\left(\sqrt{b^{d}}+\sqrt{b|I|} C_{m, k}\right)|h|+C_{m, k}|I|\|f\|_{\omega^{d-1}},
\end{aligned}
$$

where

$$
C_{m, k}=\left\{\begin{array}{lll}
(k b)^{\frac{1}{3}} & \text { if } & |m| \leq k b \\
1 & \text { if } & |m|>k b .
\end{array}\right.
$$

Proof. Some early work (cf. $[12,17,18]$ ) in this direction relies on the explicit form of Green's function which is very difficult, if not possible, to extend to more general cases. Our proof is based on an argument in [21, 10] (see also [30, 9]). More precisely, we take two test functions $v=u,(r-a) \partial_{r} u \in X$ in (3.13) successively to obtain a priori estimates without using Green's functions. In the following, $\varepsilon_{j}>$ $0(j=1, \ldots, 5)$ are some suitable real numbers.

We first take $v=u$ in (3.13). The imaginary and real parts are, respectively,

$$
\begin{gathered}
-k b^{d-1} \operatorname{Im}\left(T_{m, k}\right)|u(b)|^{2}=b^{d-1} \operatorname{Im}(h \overline{u(b)})+\operatorname{Im}(f, u)_{\omega^{d-1}}, \\
\left\|\partial_{r} u\right\|_{\omega^{d-1}}^{2}+\beta_{m}\|u\|_{\omega^{d-3}}^{2}-k^{2}\|u\|_{\omega^{d-1}}^{2}-k b^{d-1} \operatorname{Re}\left(T_{m, k}\right)|u(b)|^{2} \\
=b^{d-1} \operatorname{Re}(h \overline{u(b)})+\operatorname{Re}(f, u)_{\omega^{d-1}} .
\end{gathered}
$$

Copyright $@$ by SIAM. Unauthorized reproduction of this article is prohibited. 
In order to derive an upper bound for $\left\|\partial_{r} u\right\|_{\omega^{d-1}}^{2}+\beta_{m}\|u\|_{\omega^{d-3}}^{2}$, we proceed separately with two cases: (i) $d=2,|m|>0$ or $d=3, m \geq 0$ and (ii) $d=2, m=0$. In the first case, we have from (2.22) and (2.34b) with $|m| \geq 1$ (note that $\kappa=k b$ ) that

$$
\frac{1}{k\left|\operatorname{Re}\left(T_{m, k}\right)\right|} \leq b \quad \text { for } d=2,|m|>0 \text { or } d=3, m \geq 0 .
$$

In what follows, we shall repeatedly use the inequality $2 A B \leq \varepsilon A^{2}+\frac{B^{2}}{\varepsilon}$ for all $A, B, \varepsilon>0$.

Applying the Cauchy-Schwarz inequality to (3.17b) leads to

$$
\begin{aligned}
\left\|\partial_{r} u\right\|_{\omega^{d-1}}^{2}+ & \beta_{m}\|u\|_{\omega^{d-3}}^{2}-k b^{d-1} \operatorname{Re}\left(T_{m, k}\right)|u(b)|^{2} \\
\leq & k^{2}\|u\|_{\omega^{d-1}}^{2}+\frac{k b^{d-1}\left|\operatorname{Re}\left(T_{m, k}\right)\right|}{2}|u(b)|^{2}+\frac{b^{d-1}}{2 k\left|\operatorname{Re}\left(T_{m, k}\right)\right|}|h|^{2} \\
& +\varepsilon_{1} k^{2}\|u\|_{\omega^{d-1}}^{2}+\frac{1}{4 \varepsilon_{1} k^{2}}\|f\|_{\omega^{d-1}}^{2} .
\end{aligned}
$$

Thus, by (3.18), the estimate (3.19) becomes (for $d=2,|m|>0$ or $d=3, m \geq 0$ )

$$
\begin{aligned}
\left\|\partial_{r} u\right\|_{\omega^{d-1}}^{2} & +\beta_{m}\|u\|_{\omega^{d-3}}^{2}-\frac{k b^{d-1} \operatorname{Re}\left(T_{m, k}\right)}{2}|u(b)|^{2} \\
& \leq\left(1+\varepsilon_{1}\right) k^{2}\|u\|_{\omega^{d-1}}^{2}+\frac{b^{d}}{2}|h|^{2}+\frac{1}{4 \varepsilon_{1} k^{2}}\|f\|_{\omega^{d-1}}^{2} .
\end{aligned}
$$

To treat the only remaining case, (ii) $d=2$ and $m=0$, we apply the Cauchy-Schwarz inequality to (3.17a) and get that

$$
\begin{aligned}
k b \operatorname{Im}\left(T_{0, k}\right)|u(b)|^{2} \leq & \frac{k b \operatorname{Im}\left(T_{0, k}\right)}{2}|u(b)|^{2}+\frac{b}{2 k \operatorname{Im}\left(T_{0, k}\right)}|h|^{2} \\
& +\frac{\varepsilon_{2} k \operatorname{Im}\left(T_{0, k}\right)}{2}\|u\|_{\omega}^{2}+\frac{1}{2 \varepsilon_{2} k \operatorname{Im}\left(T_{0, k}\right)}\|f\|_{\omega}^{2}
\end{aligned}
$$

which implies that

$$
k^{2} b|u(b)|^{2} \leq \varepsilon_{2} k^{2}\|u\|_{\omega}^{2}+\frac{b}{\left|\operatorname{Im}\left(T_{0, k}\right)\right|^{2}}|h|^{2}+\frac{1}{\varepsilon_{2}\left|\operatorname{Im}\left(T_{0, k}\right)\right|^{2}}\|f\|_{\omega}^{2} .
$$

Thanks to $(2.34 \mathrm{c})$, we can rewrite the inequality (3.22) as

$$
k^{2} b|u(b)|^{2} \leq \varepsilon_{2} k^{2}\|u\|_{\omega}^{2}+b|h|^{2}+\frac{1}{\varepsilon_{2}}\|f\|_{\omega}^{2} .
$$

We now apply the Cauchy-Schwarz inequality to (3.17b) (with $d=2$ and $m=0$ ) and use (3.23) to bound the term involving $|u(b)|^{2}$ to get

$$
\begin{aligned}
\left\|\partial_{r} u\right\|_{\omega}^{2} & +\beta_{0}\|u\|_{\omega^{-1}}^{2}-k b \operatorname{Re}\left(T_{0, k}\right)|u(b)|^{2} \\
& \leq k^{2}\|u\|_{\omega}^{2}+k^{2} b|u(b)|^{2}+\frac{b}{4 k^{2}}|h|^{2}+\frac{\varepsilon_{1} k^{2}}{2}\|u\|_{\omega}^{2}+\frac{1}{2 \varepsilon_{1} k^{2}}\|f\|_{\omega}^{2} \\
& \leq\left(1+\varepsilon_{1}\right) k^{2}\|u\|_{\omega}^{2}+c b|h|^{2}+\frac{1}{\varepsilon_{1}}\|f\|_{\omega}^{2}
\end{aligned}
$$

Copyright $@$ by SIAM. Unauthorized reproduction of this article is prohibited. 
where we took $\varepsilon_{2}=\varepsilon_{1} / 2$ in (3.23). In view of (3.20) and (3.24), we have the following estimate which is valid for all cases:

$$
\left\|\partial_{r} u\right\|_{\omega^{d-1}}^{2}+\beta_{m}\|u\|_{\omega^{d-3}}^{2} \leq\left(1+\varepsilon_{1}\right) k^{2}\|u\|_{\omega^{d-1}}^{2}+c b^{d}|h|^{2}+c\|f\|_{\omega^{d-1}}^{2} .
$$

Now the main difficulty is how to bound the term $k^{2}\|u\|_{\omega^{d-1}}^{2}$. To do this, we need to derive further estimates by testing (3.13) with another function. Using a standard regularity argument, one can easily verify that for $f \in L_{\omega^{d-1}}^{2}(I)$ the weak solution of (3.13) satisfies $(r-a) \partial_{r} u \in X$. Hence, we can take $v=2(r-a) \partial_{r} u$ in (3.13), and after integration by parts and thanks to the identity

$$
(u, v)_{\omega}+(v, u)_{\omega}=2 \operatorname{Re}(u, v)_{\omega},
$$

we find that the first three terms of the real part of (3.13) with $v=2(r-a) \partial_{r} u$ are

$$
\begin{aligned}
2 \operatorname{Re}\left(\partial_{r} u, \partial_{r}\left((r-a) \partial_{r} u\right)\right)_{\omega^{d-1}} & =b^{d-1}|I|\left|\partial_{r} u(b)\right|^{2} \\
& +\int_{a}^{b}\left[(2-d)+(d-1) \frac{a}{r}\right]\left|\partial_{r} u\right|^{2} r^{d-1} d r \\
2 \beta_{m} \operatorname{Re}\left(u,(r-a) \partial_{r} u\right)_{\omega^{d-3}}= & \beta_{m} b^{d-3}|I||u(b)|^{2} \\
- & \beta_{m} \int_{a}^{b}\left[(d-2)-(d-3) \frac{a}{r}\right]|u|^{2} r^{d-3} d r \\
-2 k^{2} \operatorname{Re}\left(u,(r-a) \partial_{r} u\right)_{\omega^{d-1}} & =-k^{2} b^{d-1}|I||u(b)|^{2} \\
& +k^{2} \int_{a}^{b}\left[d-(d-1) \frac{a}{r}\right]|u|^{2} r^{d-1} d r .
\end{aligned}
$$

Accordingly, we find that the real part of (3.13) with $v=2(r-a) \partial_{r} u$ becomes

$$
\begin{aligned}
& b^{d-1}|I|\left(\left|\partial_{r} u(b)\right|^{2}+\beta_{m} b^{-2}|u(b)|^{2}\right)+a(d-1)\left\|\partial_{r} u\right\|_{\omega^{d-2}}^{2} \\
& \quad+k^{2} \int_{a}^{b}\left[d-(d-1) \frac{a}{r}\right]|u|^{2} r^{d-1} d r \\
& \leq k^{2} b^{d-1}|I||u(b)|^{2}+(d-2)\left\|\partial_{r} u\right\|_{\omega^{d-1}}^{2}+\beta_{m} \int_{a}^{b}\left[(d-2)+(3-d) \frac{a}{r}\right]|u|^{2} r^{d-3} d r \\
& \quad+2 b^{d-1}|I|\left|\operatorname{Re}\left(h \overline{\partial_{r} u(b)}\right)\right|+2\left|\operatorname{Re}\left(f,(r-a) \partial_{r} u\right)_{\omega^{d-1}}\right| .
\end{aligned}
$$

Note that in the third term the factor $d-(d-1) \frac{a}{r}>1$ for all $r \in(a, b)$, so we can use this term to bound $k^{2}\|u\|_{\omega^{d-1}}^{2}$ in (3.25).

By the Cauchy-Schwarz inequality, we can treat the last two terms at the righthand side of (3.28) as, respectively,

$$
2 b^{d-1}|I|\left|\operatorname{Re}\left(h \overline{\partial_{r} u(b)}\right)\right| \leq \frac{b^{d-1}|I|}{2}\left|\partial_{r} u(b)\right|^{2}+2 b^{d-1}|I||h|^{2},
$$

and

$$
2\left|\operatorname{Re}\left(f,(r-a) \partial_{r} u\right)_{\omega^{d-1}}\right| \leq \varepsilon_{3}\left\|\partial_{r} u\right\|_{\omega^{d-1}}^{2}+\frac{|I|^{2}}{\varepsilon_{3}}\|f\|_{\omega^{d-1}}^{2} .
$$

We now proceed separately for $d=2$ and $d=3$.

Copyright $@$ by SIAM. Unauthorized reproduction of this article is prohibited. 
Case I: $d=2$. In this case, a combination of (3.28)-(3.30) leads to

$$
\begin{aligned}
b|I|\left(\left|\partial_{r} u(b)\right|^{2}+\right. & \left.\beta_{m} b^{-2}|u(b)|^{2}\right)+a\left\|\partial_{r} u\right\|^{2}+k^{2} \int_{a}^{b}\left[2-\frac{a}{r}\right]|u|^{2} r d r \\
\leq & k^{2} b|I \| u(b)|^{2}+\left(\varepsilon_{3}\left\|\partial_{r} u\right\|_{\omega}^{2}+a \beta_{m}\|u\|_{\omega^{-2}}^{2}\right)+\frac{b|I|}{2}\left|\partial_{r} u(b)\right|^{2} \\
& +2 b|I||h|^{2}+\frac{|I|^{2}}{\varepsilon_{3}}\|f\|_{\omega}^{2} .
\end{aligned}
$$

Using (3.25) with $d=2$, we have that, for $\varepsilon_{3}<1$ and for certain $\xi_{1} \in(a, b)$,

$$
\begin{gathered}
\varepsilon_{3}\left\|\partial_{r} u\right\|_{\omega}^{2}+a \beta_{m}\|u\|_{\omega^{-2}}^{2} \leq \max \left\{\varepsilon_{3}, \frac{a}{\xi_{1}}\right\}\left(\left\|\partial_{r} u\right\|_{\omega}^{2}+\beta_{m}\|u\|_{\omega^{-1}}^{2}\right) \\
\leq\left(1+\varepsilon_{1}\right) k^{2}\|u\|_{\omega}^{2}+c b^{2}|h|^{2}+c\|f\|_{\omega}^{2} .
\end{gathered}
$$

Hence, it remains to bound the term $k^{2} b|I||u(b)|^{2}$ in (3.31).

(i) $|m|>k b$. In this case, the term $b|I| k^{2}|u(b)|^{2}$ can be absorbed by $b^{-1}|I| \beta_{m}|u(b)|^{2}$ at the left-hand side of (3.31). Hence, a combination of (3.31)-(3.32) leads to the desired result:

$$
\begin{aligned}
b|I|\left(\frac{1}{2}\left|\partial_{r} u(b)\right|^{2}\right. & \left.+\left(\beta_{m} b^{-2}-k^{2}\right)|u(b)|^{2}\right)+a\left\|\partial_{r} u\right\|^{2}+C k^{2}\|u\|_{\omega}^{2} \\
& \lesssim b^{2}|h|^{2}+\left(1+|I|^{2}\right)\|f\|_{\omega}^{2},
\end{aligned}
$$

where, with a suitable choice of $\varepsilon_{1}$, the constant

$$
C=1-\frac{a}{\xi_{2}}-\varepsilon_{1}>0 \quad \text { for certain } \xi_{2} \in(a, b) .
$$

(ii) $|m| \leq k b$. Similar to the derivation of (3.22), we apply the Cauchy-Schwarz inequality to $(3.17 \mathrm{a})$ :

$$
k^{2} b|I||u(b)|^{2} \leq \varepsilon_{3}|I| k^{2}\|u\|_{\omega}^{2}+\frac{b|I|}{\left|\operatorname{Im}\left(T_{m, k}\right)\right|^{2}}|h|^{2}+\frac{|I|}{\varepsilon_{3}\left|\operatorname{Im}\left(T_{m, k}\right)\right|^{2}}\|f\|_{\omega}^{2} .
$$

Then a combination of the estimates (3.31), (3.32), and (3.35) leads to

$$
\begin{aligned}
b|I|\left(\frac{1}{2}\left|\partial_{r} u(b)\right|^{2}\right. & \left.+\beta_{m} b^{-2}|u(b)|^{2}\right)+a\left\|\partial_{r} u\right\|^{2}+\widetilde{C} k^{2}\|u\|_{\omega}^{2} \\
& \lesssim C_{m, k}^{(1)}|h|^{2}+C_{m, k}^{(2)}\|f\|_{\omega}^{2},
\end{aligned}
$$

where, with a suitable choice of $\varepsilon_{1}$ and $\varepsilon_{3}$ and using the fact that $\operatorname{Im}\left(T_{m, k}\right)<$ 1 , the constants are

$$
\begin{aligned}
& \widetilde{C}=1-\frac{a}{\xi_{3}}-\varepsilon_{1}-\varepsilon_{3}|I|>0 \quad \text { for certain } \xi_{3} \in(a, b), \\
& C_{m, k}^{(1)}=b^{2}+\frac{b|I|}{\left|\operatorname{Im}\left(T_{m, k}\right)\right|^{2}}, \\
& C_{m, k}^{(2)}=|I|^{2}+\frac{|I|}{\varepsilon_{3}\left|\operatorname{Im}\left(T_{m, k}\right)\right|^{2}} \lesssim|I|^{2}\left(1+\frac{1}{\left|\operatorname{Im}\left(T_{m, k}\right)\right|^{2}}\right) .
\end{aligned}
$$

Notice that we have

$$
\operatorname{Im}\left(T_{m, k}\right) \geq c(k b)^{-\frac{1}{3}} \quad \text { for }|m| \leq k b,
$$

since $\operatorname{Im}\left(T_{m, k}\right)$ is a decreasing function of $m$ and the estimate (2.35).

Copyright $@$ by SIAM. Unauthorized reproduction of this article is prohibited. 
Therefore, the desired result (3.15) with $d=2$ follows from (3.25), (3.33), and (3.36).

Case II: $d=3$. In this case, a combination of (3.28)-(3.30) leads to

$$
\begin{aligned}
b^{2}|I|\left(\left|\partial_{r} u(b)\right|^{2}+\right. & \left.\beta_{m} b^{-2}|u(b)|^{2}\right)+2 a\left\|\partial_{r} u\right\|_{\omega}^{2}+k^{2} \int_{a}^{b}\left[3-\frac{2 a}{r}\right]|u|^{2} r d r \\
\leq & k^{2} b^{2}|I||u(b)|^{2}+\frac{b^{2}|I|}{2}\left|\partial_{r} u(b)\right|^{2}+\left(\left\|\partial_{r} u\right\|_{\omega^{2}}^{2}+\beta_{m}\|u\|^{2}\right) \\
& +2 b^{2}|I||h|^{2}+\frac{|I|^{2}}{\varepsilon_{3}}\|f\|_{\omega^{2}}^{2} .
\end{aligned}
$$

By (3.25),

$$
\left\|\partial_{r} u\right\|_{\omega^{2}}^{2}+\beta_{m}\|u\|^{2} \leq\left(1+\varepsilon_{1}\right) k^{2}\|u\|_{\omega^{2}}^{2}+c b^{3}|h|^{2}+c\|f\|_{\omega^{2}}^{2} .
$$

The rest of the proof is essentially the same as that in the 2-D case. More precisely, we can derive the 3-D version of inequalities (3.33)-(3.38) with slightly different constants

$$
\begin{aligned}
& C=2-\frac{2 a}{\xi_{3}}-\varepsilon_{1}>0, \quad \xi_{3} \in(a, b), \quad \text { if } m \geq k b, \\
& \tilde{C}=2-\frac{2 a}{\xi_{3}}-\varepsilon_{1}-2 \varepsilon_{3}|I|, \quad \xi_{3} \in(a, b), \quad \text { if } m<k b .
\end{aligned}
$$

Finally, since $\operatorname{Im}\left(T_{m, k}\right)$ is a decreasing function of $m$ and $\operatorname{Im}\left(T_{m, k}\right)=\operatorname{Im}\left(\mathcal{T}_{m, k b}\right)$ (cf. (2.14) and (3.7)), the desired bound follows from (2.27) and (2.35).

The proof of Theorem 3.1. Since the proof of the 2-D and 3-D cases is essentially the same, we prove only (3.12) with $d=3$. Thanks to the orthogonality of the spherical harmonic functions, we deduce from Lemma 3.1 that

$$
\begin{aligned}
\|\nabla U\|^{2} & +k^{2}\|U\|^{2} \lesssim\left\|\partial_{r} U\right\|_{\omega^{2}}^{2}+\left\|\nabla_{S} U\right\|^{2}+k^{2}\|U\|_{\omega^{2}}^{2} \\
& \lesssim \sum_{m=0}^{\infty} \sum_{l=-m}^{m}\left(\left\|\partial_{r} \hat{u}_{l m}\right\|_{\omega^{2}}^{2}+\beta_{m}\left\|\hat{u}_{l m}\right\|^{2}+k^{2}\left\|\hat{u}_{l m}\right\|_{\omega^{2}}^{2}\right) \\
& \lesssim \sum_{m=0}^{\infty} \sum_{l=-m}^{m}\left(\left(\sqrt{b^{3}}+\sqrt{b|I|} C_{m, k}\right)^{2}\left|\hat{h}_{l m}\right|^{2}+C_{m, k}^{2}|I|^{2}\left\|\hat{f}_{l m}\right\|_{\omega^{2}}^{2}\right) \\
& \lesssim \sum_{m=0}^{\infty} \sum_{l=-m}^{m}\left(\left(\sqrt{b^{3}}+\sqrt{b|I|}(k b)^{1 / 3}\right)^{2} \hat{h}_{l m}^{2}+(k b)^{2 / 3}|I|^{2}\left\|\hat{f}_{l m}\right\|_{\omega^{2}}^{2}\right) \\
& \lesssim\left(\sqrt{b^{3}}+\sqrt{b|I|}(k b)^{1 / 3}\right)^{2}\|\eta\|_{L^{2}(S)}+(k b)^{2 / 3}|I|^{2}\|F\|^{2} .
\end{aligned}
$$

This ends the proof.

\section{Spectral-Galerkin approximation.}

4.1. The spectral-Galerkin method and its well-posedness. Let $P_{N}$ be the space of all complex polynomials of degree at most $N$ on $\bar{I}$. Define $X_{N}:=\{u \in$ $\left.P_{N}: u(a)=0\right\}$ and

$$
Y_{M}:= \begin{cases}\operatorname{span}\left\{e^{\mathrm{i} m \theta}:-M \leq m \leq M\right\} & \text { if } d=2, \\ \operatorname{span}\left\{Y_{m}^{l}(\theta, \phi): 0 \leq|l| \leq m \leq M\right\} & \text { if } d=3,\end{cases}
$$

where $\mathcal{B}(\cdot, \cdot)$ is defined in $(3.9)$.

Copyright (c) by SIAM. Unauthorized reproduction of this article is prohibited. 
The spectral-Galerkin approximation to (3.11) is as follows:

Find $U_{M N} \in \mathcal{V}_{M N}:=X_{N} \times Y_{M}$ such that

$$
\mathcal{B}\left(U_{M N}, V_{M N}\right)=\left(F, V_{M N}\right)_{\omega^{d-1}}+b^{d-1}\left\langle\eta, V_{M N}(b, \cdot)\right\rangle_{S} \quad \forall V_{M N} \in \mathcal{V}_{M N} .
$$

Since the sesquilinear form $\mathcal{B}(\cdot, \cdot)$ is not coercive in $\mathcal{V}_{M N} \times \mathcal{V}_{M N}$ even for small wave number $k$, an important issue is to prove the well-posedness of the discrete scheme (4.4).

Expanding the numerical solution and test function as

$$
\left(U_{M N}, V_{M N}\right)= \begin{cases}\sum_{|m|=0}^{M}\left(\hat{u}_{m}^{N}(r), \hat{v}_{m}^{N}(r)\right) e^{\mathrm{i} m \theta} & \text { if } d=2, \\ \sum_{m=0}^{M} \sum_{l=-m}^{m}\left(\hat{u}_{l m}^{N}(r), \hat{v}_{l m}^{N}(r)\right) Y_{m}^{l}(\theta, \phi) & \text { if } d=3,\end{cases}
$$

one verifies that $u_{N}:=\hat{u}_{m}^{N}$ or $\hat{u}_{l m}^{N}$ satisfies the reduced problem

$$
\left\{\begin{array}{l}
\text { Find } u_{N} \in X_{N} \text { such that } \\
\mathcal{B}_{m}\left(u_{N}, v_{N}\right)=\left(f, v_{N}\right)_{\omega^{d-1}}+b^{d-1} h \overline{v_{N}(b)} \quad \forall v_{N} \in X_{N}, \quad d=2,3
\end{array}\right.
$$

where $\mathcal{B}_{m}(\cdot, \cdot)$ is defined in (3.14); for brevity, we denote $v_{N}:=\hat{v}_{m}^{N}$ or $v_{l m}^{N}$, and $f$ and $h$ are the same as those in (3.13).

It is important to note that, unlike in the Galerkin finite-element method, the spectral-Galerkin approximation space $X_{N}$ has the following property: For $u_{N} \in X_{N}$, we have $(r-a) \partial_{r} u_{N} \in X_{N}$. Hence, the proof of Lemma 3.1 is also valid for the discrete system (4.4). In particular, Theorem 3.1 holds with $u_{N}$ in the place of $u$. As a consequence, the problem (4.4) has at most one solution. Since (4.4) is finitedimensional, we then derive from a simple fact in linear algebra that the problem (4.4) admits a unique solution.

Therefore, following the same procedure as in the proof of Theorem 3.1 leads to the following result.

THEOREM 4.1. If $F \in L^{2}(\Omega)$ and $\eta \in L^{2}(S)$, the problem (4.2) admits a unique solution satisfying

$$
\left\|\nabla U_{M N}\right\|+k\left\|U_{M N}\right\| \lesssim\left(\sqrt{b^{d}}+\sqrt{b|I|}(k b)^{1 / 3}\right)\|\eta\|_{L^{2}(S)}+(k b)^{1 / 3}|I|\|F\| .
$$

4.2. Error estimates. In this part, we shall estimate the error between $U$ (solution of (3.11)) and $U_{M N}$ (solution of (4.2)). Our starting point is to analyze the error of 1-dimensional approximation (4.4).

4.2.1. Analysis of the 1-D scheme. In order to carry out the error analysis, we define the orthogonal projection ${ }_{0} \pi_{N}^{1}: X \rightarrow X_{N}$ by

$$
\left(\partial_{r}\left(u-{ }_{0} \pi_{N}^{1} u\right), \partial_{r} v_{N}\right)=0 \quad \forall v_{N} \in X_{N} .
$$

For $s \geq 1$ and $s \in \mathbb{N}$, we introduce the weighted Sobolev space

$$
B^{s}(I):=\left\{u \in L^{2}(I):[(r-a)(b-r)]^{\frac{l-1}{2}} \partial_{r}^{l} u \in L^{2}(I), 1 \leq l \leq s\right\},
$$

with the norm and seminorm

$$
\begin{aligned}
& \|u\|_{B^{s}}=\left(\|u\|^{2}+\sum_{l=1}^{s}\left\|[(r-a)(b-r)]^{\frac{l-1}{2}} \partial_{r}^{l} u\right\|^{2}\right)^{\frac{1}{2}}, \\
& |u|_{B^{s}}=\left\|[(r-a)(b-r)]^{\frac{s-1}{2}} \partial_{r}^{s} u\right\| .
\end{aligned}
$$

Copyright $@$ by SIAM. Unauthorized reproduction of this article is prohibited. 
Lemma 4.1. For any $u \in X \cap B^{s}(I)$, with $s \geq 1$ and $s \in \mathbb{N}$,

$$
\left\|\partial_{r}\left({ }_{0} \pi_{N}^{1} u-u\right)\right\|+N|I|^{-1}\left\|_{0} \pi_{N}^{1} u-u\right\| \lesssim N^{1-s}|u|_{B^{s}} .
$$

Proof. This result is a direct consequence of the Legendre polynomial approximation (with a scaling and a direct extension to complex functions), which can be found, for instance, in [7], with an improvement of the weighted seminorm in the upper bound given by [14].

With the aid of Lemmas 3.1 and 4.1, we are able to obtain the following error estimates.

THEOREM 4.2. Let $u$ and $u_{N}$ be, respectively, the solutions of (3.13) and (4.4). If $u \in X \cap B^{s}(I)$, with integer $s \geq 1$, then for $d=2,3$

$$
\begin{aligned}
\left\|\partial_{r}\left(u-u_{N}\right)\right\|_{\omega^{d-1}} & +\sqrt{\beta_{m}}\left\|u-u_{N}\right\|_{\omega^{d-3}}+k\left\|u-u_{N}\right\|_{\omega^{d-1}} \\
& \lesssim C_{\star}(m, N, k ; a, b, d) N^{1-s}|u|_{B^{s}},
\end{aligned}
$$

where

$$
\begin{aligned}
C_{\star}(m, N, k ; a, b, d): & =\left(1+\sqrt{\beta_{m}}\right) b^{(d-1) / 2}+\sqrt{\beta_{m}} a^{\frac{d-3}{2}}|I| N^{-1} \\
& +k^{1 / 3}\left(\sqrt{\beta_{m}} b^{3 d / 2-2} \sqrt{|I|} N^{-1 / 2}+|I|^{2} b^{d / 2} k^{2} N^{-1}\right) .
\end{aligned}
$$

Proof. Let $e_{N}=u_{N}-{ }_{0} \pi_{N}^{1} u$ and $\tilde{e}_{N}=u-{ }_{0} \pi_{N}^{1} u$. By (3.13) and (4.4),

$$
\mathcal{B}_{m}\left(u-u_{N}, v_{N}\right)=0 \quad \forall v_{N} \in X_{N} .
$$

Then we derive from (3.14), (4.6), and (4.10) that for any $v_{N} \in X_{N}$

$$
\begin{aligned}
\mathcal{B}_{m}\left(e_{N}, v_{N}\right)= & \mathcal{B}_{m}\left(\tilde{e}_{N}, v_{N}\right)=\beta_{m}\left(\tilde{e}_{N}, v_{N}\right)_{\omega^{d-3}} \\
& -k^{2}\left(\tilde{e}_{N}, v_{N}\right)_{\omega^{d-1}}-k b^{d-1} T_{m, k} \tilde{e}_{N}(b) \overline{v_{N}(b)} .
\end{aligned}
$$

Hence, we can view (4.11) in the form of (3.13) with $u=e_{N}, h=-k b^{d-1} T_{m, k} \tilde{e}_{N}(b)$, $f=-k^{2} \tilde{e}_{N}$, and an additional term $\beta_{m}\left(\tilde{e}_{N}, v_{N}\right)_{\omega^{d-3}}$. As with the proof of Theorem 3.1 , we take two different test functions $v_{N}=e_{N}, 2(r-a) \partial_{r} e_{N} \in X_{N}$ and treat the extra term as

$$
\beta_{m}\left|\left(\tilde{e}_{N}, e_{N}\right)_{\omega^{d-3}}\right| \leq \varepsilon_{6} \beta_{m}\left\|e_{N}\right\|_{\omega^{d-3}}^{2}+\frac{\beta_{m}}{4 \varepsilon_{6}}\left\|\tilde{e}_{N}\right\|_{\omega^{d-3}}^{2}
$$

and

$$
\begin{aligned}
& 2 \beta_{m}\left|\left(\tilde{e}_{N},(r-a) \partial_{r} e_{N}\right)_{\omega^{d-3}}\right| \leq 2 \beta_{m}\left\{b^{d-3}|I|\left|\tilde{e}_{N}(b) \overline{e_{N}(b)}\right|\right. \\
& \left.\quad+\left|\left(\partial_{r} \tilde{e}_{N},\left(1-a r^{-1}\right) e_{N}\right)_{\omega^{d-2}}\right|+\left|\left(\tilde{e}_{N},\left((d-2)-a(d-3) r^{-1}\right) e_{N}\right)_{\omega^{d-3}}\right|\right\} \\
& \leq \varepsilon_{7} \beta_{m} b^{d-3}|I|\left|e_{N}(b)\right|^{2}+\frac{\beta_{m} b^{d-3}|I|}{\varepsilon_{7}}\left|\tilde{e}_{N}(b)\right|^{2}+\varepsilon_{8} \beta_{m}\left\|e_{N}\right\|_{\omega^{d-3}}^{2} \\
& \quad+\frac{c \beta_{m}}{\varepsilon_{8}}\left(\left\|\partial_{r} \tilde{e}_{N}\right\|_{\omega^{d-1}}^{2}+\left\|\tilde{e}_{N}\right\|_{\omega^{d-3}}^{2}\right) .
\end{aligned}
$$

Thus, choosing suitable constants $\left\{\varepsilon_{j}\right\}_{j=6}^{8}$ and following the same lines as for the proof of Theorem 3.1 (with $u=e_{N}, h=-k b^{d-1} T_{m, k} \tilde{e}_{N}(b)$, and $f=-k^{2} \tilde{e}_{N}$ ), we can 
derive that

$$
\begin{aligned}
& \left\|\partial_{r} e_{N}\right\|_{\omega^{d-1}}^{2}+\beta_{m}\left\|e_{N}\right\|_{\omega^{d-3}}^{2}+k^{2}\left\|e_{N}\right\|_{\omega^{d-1}}^{2} \\
& \quad \lesssim \beta_{m}\left(\left\|\partial_{r} \tilde{e}_{N}\right\|_{\omega^{d-1}}^{2}+\left\|\tilde{e}_{N}\right\|_{\omega^{d-3}}^{2}\right)+\beta_{m} b^{d-3}|I|\left|\tilde{e}_{N}(b)\right|^{2} \\
& \quad+k^{2} b^{2(d-1)}\left|T_{m, k}\right|^{2}\left(\sqrt{b^{d}}+\sqrt{b|I|} C_{m, k}\right)^{2}\left|\tilde{e}_{N}(b)\right|^{2} \\
& \quad+k^{4}|I|^{2} C_{m, k}^{2}\left\|\tilde{e}_{N}\right\|_{\omega^{d-1}}^{2} .
\end{aligned}
$$

To estimate the term $\left|\tilde{e}_{N}(b)\right|$, we use the Sobolev inequality and Lemma 4.1 to obtain that

$$
\left|\tilde{e}_{N}(b)\right|^{2} \lesssim\left(2+|I|^{-1}\right)\left\|\tilde{e}_{N}\right\|\left\|\tilde{e}_{N}\right\|_{1} \lesssim N^{1-2 s}|I||u|_{B^{s}}^{2} .
$$

Next, using the inequality $\|v\|_{\omega^{\alpha}}^{2} \leq \max \left\{b^{\alpha}, a^{\alpha}\right\}\|v\|^{2}$ and Lemma 4.1 leads to

$$
\begin{aligned}
& \left\|\partial_{r}^{\mu} \tilde{e}_{N}\right\|_{\omega^{d-1}}^{2} \leq b^{d-1}\left\|\partial_{r}^{\mu} \tilde{e}_{N}\right\|^{2} \lesssim b^{d-1}|I|^{2-2 \mu} N^{2 \mu-2 s}|u|_{B^{s}}^{2}, \quad \mu=0,1, \\
& \left\|\tilde{e}_{N}\right\|_{\omega^{d-3}}^{2} \leq a^{d-3}\left\|\tilde{e}_{N}\right\|^{2} \lesssim a^{d-3}|I|^{2} N^{-2 s}|u|_{B^{s}}^{2} .
\end{aligned}
$$

Hence, by the triangle inequality, (4.14)-(4.16), and Lemma 4.1, we have that

$$
\begin{aligned}
&\left\|\partial_{r}\left(u-u_{N}\right)\right\|_{\omega^{d-1}}^{2}+\beta_{m}\left\|u-u_{N}\right\|_{\omega^{d-3}}^{2}+k^{2}\left\|u-u_{N}\right\|_{\omega^{d-1}}^{2} \\
& \leq\left(\left\|\partial_{r} e_{N}\right\|_{\omega^{d-1}}^{2}+\beta_{m}\left\|e_{N}\right\|_{\omega^{d-3}}^{2}+k^{2}\left\|e_{N}\right\|_{\omega^{d-1}}^{2}\right) \\
&+\left(\left\|\partial_{r} \tilde{e}_{N}\right\|_{\omega^{d-1}}^{2}+\beta_{m}\left\|\tilde{e}_{N}\right\|_{\omega^{d-3}}^{2}+k^{2}\left\|\tilde{e}_{N}\right\|_{\omega^{d-1}}^{2}\right) \\
& \lesssim\left(1+\beta_{m}\right)\left\|\partial_{r} \tilde{e}_{N}\right\|_{\omega^{d-1}}^{2}+\beta_{m}\left\|\tilde{e}_{N}\right\|_{\omega^{d-3}}^{2}+\beta_{m} b^{d-3}\left|I \| \tilde{e}_{N}(b)\right|^{2} \\
&+k^{2} b^{2(d-1)}\left|T_{m, k}\right|^{2}\left(\sqrt{b^{d}}+\sqrt{b|I|} C_{m, k}\right)^{2}\left|\tilde{e}_{N}(b)\right|^{2} \\
&+k^{4}|I|^{2} C_{m, k}^{2}\left\|\tilde{e}_{N}\right\|_{\omega^{d-1}}^{2} \\
& \lesssim C^{*}(m, N, k ; a, b, d) N^{2-2 s}|u|_{B^{s}}^{2},
\end{aligned}
$$

where

$$
\begin{aligned}
& C^{*}(m, N, k ; a, b, d):=\left(1+\beta_{m}\right) b^{d-1}+\beta_{m} a^{d-3}|I|^{2} N^{-2} \\
& \quad+\beta_{m} b^{d-3}|I|^{2} N^{-1}+k^{2} b^{2(d-1)}\left|T_{m, k}\right|^{2}\left(\sqrt{b^{d}}+\sqrt{b|I|} C_{m, k}\right)^{2}|I| N^{-1} \\
& \quad+k^{4}|I|^{4} b^{d-1} C_{m, k}^{2} N^{-2} .
\end{aligned}
$$

We now derive an upper bound for $C^{*}(m, N, k ; a, b, d)$. Since by (2.22) and (2.34)

$$
\left|T_{m, k}\right|^{2} \leq 1+\frac{(m+1)^{2}}{(k b)^{2}}
$$

and by $(3.16) C_{m, k} \lesssim(k b)^{1 / 3}$, we deduce that for $N \gg 1$

$$
\begin{aligned}
C^{*}(m, N, k ; a, b, d) & \lesssim\left(1+\beta_{m}\right) b^{d-1}+\beta_{m} a^{d-3}|I|^{2} N^{-2} \\
& +\beta_{m} b^{3 d-4}|I| k^{2 / 3} N^{-1}+|I|^{4} b^{d} k^{4+2 / 3} N^{-2} .
\end{aligned}
$$

This implies the desired result.

Remark 4.1. Note that, in the error estimate (4.8), $N^{1-s}|u|_{B^{s}}$ is the best approximation error, and $k^{2} N^{-1}$ in $C_{*}$ is the so-called "pollution error" which is typical for the numerical approximations to the Helmholtz equation (cf. [4]). The extra term

Copyright (c) by SIAM. Unauthorized reproduction of this article is prohibited. 
$k^{1 / 3}$ in $C_{*}$ is due to the asymptotic behavior of the DtN kernel (see section 2), and it is unlikely that this extra term can be removed.

Remark 4.2. To illustrate how the error behaves with respect to $N, k$, and $b$ with $a>0$ being fixed, we consider a typical oscillatory function $u(r)=e^{i k r}-e^{i k a}$. Then, for any $s>0$, we have

$$
\begin{aligned}
|u|_{B^{s}}^{2} & =\int_{a}^{b}\left|\partial_{r}^{s} u\right|^{2}((r-a)(b-r))^{s-1} d r \\
& \leq k^{2 s} \int_{a}^{b}((r-a)(b-r))^{s-1} d r \lesssim k\left(k \frac{b-a}{2}\right)^{2 s-1} .
\end{aligned}
$$

Plugging this into (4.8), we find that for this particular but typical solution we have that for any $s \geq 1$

$$
\begin{aligned}
\left\|\partial_{r}\left(u-u_{N}\right)\right\|_{\omega^{d-1}} & +\sqrt{\beta_{m}}\left\|u-u_{N}\right\|_{\omega^{d-3}}+k\left\|u-u_{N}\right\|_{\omega^{d-1}} \\
& \lesssim C_{\star}(m, N, k ; a, b, d) k \sqrt{\frac{b-a}{2}}\left(\frac{k(b-a)}{2 N}\right)^{1-s} .
\end{aligned}
$$

Hence, the error will decay exponentially as soon as $\frac{k(b-a)}{2 N}<1$, as opposed to the usual condition $\frac{k b}{2 N}<1$. Hence, we can significantly reduce the computational cost by choosing $b$ as close to $a$ as we wish (note, however, that, for scattering from a general obstacle $D=r>a+g(\theta)$ in 2-D or $D=r>a+g(\theta, \phi)$ in 3-D, we have to make sure that $\left.b>a+\|g\|_{L^{\infty}}\right)$.

With the above preparations, we are ready to perform the error analysis of the full scheme (4.2).

4.2.2. Multidimensional cases. To describe the error, we introduce the following nonisotropic Sobolev space:

$$
\mathcal{H}_{p, \omega^{d-1}}^{s, s^{\prime}}(\Omega)=L_{p}^{2}\left(S ; B^{s}(I)\right) \cap H_{p}^{s^{\prime}-1}\left(S ; H_{\omega^{d-1}}^{1}(I)\right) \cap H_{p}^{s^{\prime}}\left(S ; L_{\omega^{d-3}}^{2}(I) \cap L_{\omega^{d-1}}^{2}(I)\right),
$$

with $d=2,3, s, s^{\prime} \geq 1$, and the norm

$$
\begin{aligned}
\|U\|_{\mathcal{H}_{p, \omega}^{s, s^{\prime}}(\Omega)}= & \left(\sum _ { | m | = 0 } ^ { \infty } \left[\left|\hat{u}_{m}\right|_{B^{s}}^{2}+\left(1+m^{2}\right)^{s^{\prime}-1}\left\|\partial_{r} \hat{u}_{m}\right\|_{\omega}^{2}\right.\right. \\
& \left.\left.+\left(1+m^{2}\right)^{s^{\prime}}\left(\left\|\hat{u}_{m}\right\|_{\omega^{-1}}^{2}+\left\|\hat{u}_{m}\right\|_{\omega}^{2}\right)\right]\right)^{\frac{1}{2}} ; \\
\|U\|_{\mathcal{H}_{p, \omega^{2}}^{s, s^{\prime}}(\Omega)}= & \left(\sum _ { m = 0 } ^ { \infty } \sum _ { l = - m } ^ { m } \left[\left|\hat{u}_{l m}\right|_{B^{s}}^{2}+(1+m)^{2 s^{\prime}-s}\left\|\partial_{r} \hat{u}_{l m}\right\|_{\omega^{2}}^{2}\right.\right. \\
& \left.\left.+(1+m)^{2 s^{\prime}}\left(\left\|\hat{u}_{l m}\right\|^{2}+\left\|\hat{u}_{l m}\right\|_{\omega^{2}}^{2}\right)\right]\right)^{\frac{1}{2}} .
\end{aligned}
$$

THEOREM 4.3. Let $U$ and $U_{M N}$ be the solutions of (3.11) and (4.2), respectively. If $U \in L_{p}^{2}(S ; X) \cap \mathcal{H}_{p, \omega^{d-1}}^{s, s^{\prime}}(\Omega)$, with $d=2,3$ and $s, s^{\prime} \geq 1$, then we have

$$
\begin{aligned}
\| \nabla(U & \left.-U_{M N}\right)\|+k\| U-U_{M N} \| \\
& \lesssim\left(C_{*}(M, N, k ; a, b, d) N^{1-s}+\left(1+k M^{-1}\right) M^{1-s^{\prime}}\right)\|U\|_{\mathcal{H}_{p, \omega^{d-1}}^{s, s^{\prime}}(\Omega)},
\end{aligned}
$$

Copyright $@$ by SIAM. Unauthorized reproduction of this article is prohibited. 
where

$$
\begin{aligned}
C_{\star}(M, N, k ; a, b, d): & =(1+M) b^{(d-1) / 2}+M a^{\frac{d-3}{2}}|I| N^{-1} \\
& +k^{1 / 3}\left(M b^{3 d / 2-2} \sqrt{|I|} N^{-1 / 2}+|I|^{2} b^{d / 2} k^{2} N^{-1}\right) .
\end{aligned}
$$

Proof. Since the proof of $d=2,3$ is quite similar, we shall prove only the case $d=2$. For notational convenience, let $E_{M N}=U-U_{M N}$ and $\hat{e}_{m}=\hat{u}_{m}-\hat{u}_{m}^{N}$. Thanks to the orthogonality of the Fourier series, we have that

$$
\begin{aligned}
& \left\|\nabla E_{M N}\right\|^{2}+k^{2}\left\|E_{M N}\right\|^{2} \lesssim \sum_{|m|=0}^{M}\left(\left\|\partial_{r} \hat{e}_{m}\right\|_{\omega}^{2}+m^{2}\left\|\hat{e}_{m}\right\|_{\omega^{-1}}^{2}\right. \\
& \left.\quad+k^{2}\left\|\hat{e}_{m}\right\|_{\omega}^{2}\right)+\sum_{|m|>M}\left(\left\|\partial_{r} \hat{u}_{m}\right\|_{\omega}^{2}+m^{2}\left\|\hat{u}_{m}\right\|_{\omega^{-1}}^{2}+k^{2}\left\|\hat{u}_{m}\right\|_{\omega}^{2}\right):=S_{1}^{2}+S_{2}^{2} .
\end{aligned}
$$

Using Theorem 4.2 leads to

$$
\begin{aligned}
S_{1} & \lesssim\left(\max _{0 \leq|m| \leq M}\left\{C_{\star}(m, \ldots)\right\}\right) N^{1-s}\left(\sum_{|m|=0}^{M}\left|\hat{u}_{m}\right|_{B^{s}}^{2}\right)^{\frac{1}{2}} \\
& \lesssim C_{\star}(M, N, k ; a, b, d) N^{1-s}\|U\|_{\mathcal{H}_{p, \omega}^{s, s^{\prime}}(\Omega)}
\end{aligned}
$$

We treat $S_{2}$ as

$$
\begin{aligned}
S_{2} \lesssim & M^{1-s^{\prime}}\left(\sum_{|m|>M} m^{2 s^{\prime}-2}\left(\left\|\partial_{r} \hat{u}_{m}\right\|_{\omega}^{2}+m^{2}\left\|\hat{u}_{m}\right\|_{\omega-1}^{2}\right)\right)^{\frac{1}{2}} \\
& +k M^{-s^{\prime}}\left(\sum_{|m|>M} m^{2 s^{\prime}}\left\|\hat{u}_{m}\right\|_{\omega}^{2}\right)^{\frac{1}{2}} \\
& \lesssim\left(1+k M^{-1}\right) M^{1-s^{\prime}}\|U\|_{\mathcal{H}_{p, \omega}^{s, s^{\prime}}(\Omega)} .
\end{aligned}
$$

Hence, a combination of (4.25)-(4.27) yields the desired result.

5. Numerical results and discussions. We now present some numerical results to complement our error estimates for the spectral-Galerkin scheme (4.2). We consider the problem (3.1) in 2-D and take

$$
F(r, \theta)=0, \quad \eta(\theta)=0, \quad \xi(\theta)=H_{m}^{(1)}(k a) e^{i m \theta}
$$

In this case the exact solution is $U(r, \theta)=H_{m}^{(1)}(k r) e^{i m \theta}$. Since for a given $m$, $e^{i m \theta} \operatorname{can}$ be exactly determined with the number of mode $M=N_{\theta} \geq 2 \mathrm{~m}$, we will concentrate on the approximation behavior of our scheme with respect to the frequency $k$ and the thickness of the annulus $b-a$.

In the first set of tests, we take $a=1$ and $b=2$. In Figure 5.1, we present the relative $L^{2}$-error versus the number of mode $N=N_{r}$ for a wide range of wave numbers. We note that, as soon as $N_{r}>k(b-a) / 2$, the errors start to decay, for moderate to large wave numbers, the errors decay slowly until about $N_{r} \sim k(b-a)$, and finally, for $N_{r}>k(b-a)$, all errors converge to zero at an exponential rate.

In the second set of tests, we take $a=1$ and $b=1.25$. The results are plotted in Figure 5.2. We observe similar behaviors as in the first set except that now we have 

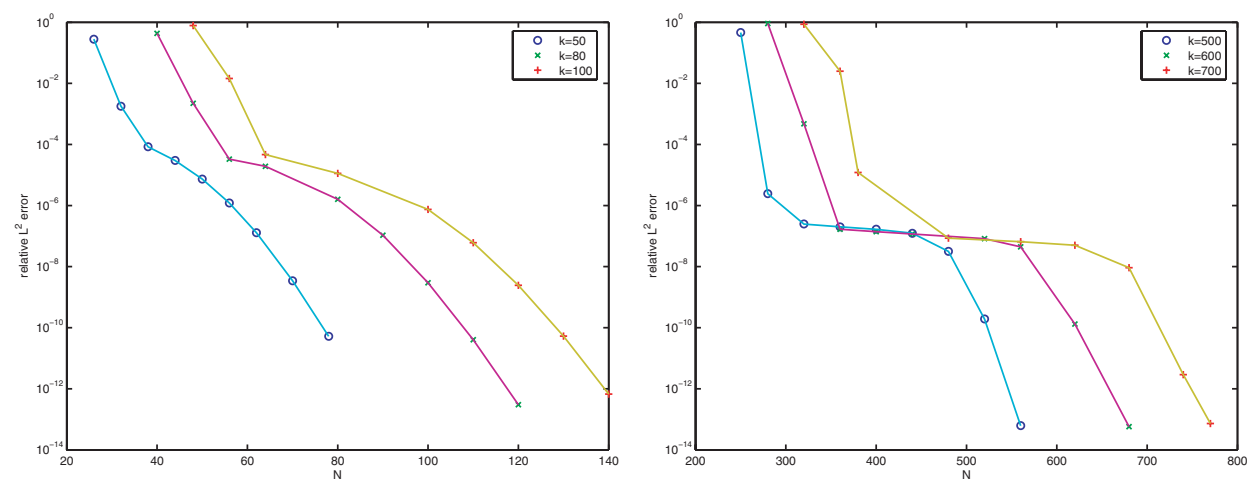

FIG. 5.1. Relative $L^{2}$-error versus $N_{r}$ as compared to an exact solution: $a=1, b=2$.
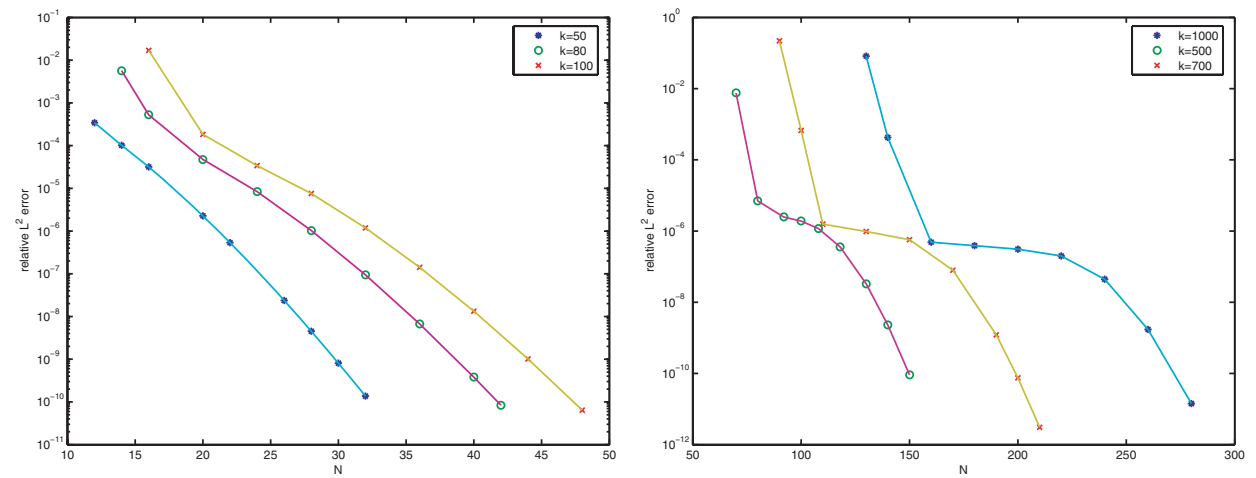

FIG. 5.2. Relative $L^{2}$-error versus $N_{r}$ as compared to an exact solution: $a=1, b=1.25$.

$b-a=\frac{1}{4}$ and only about $1 / 4$ of the modes are needed to achieve a similar accuracy. These behaviors are consistent with our error estimates (cf. Remark 4.2).

These results indicate that (i) the approximate solution $U_{N_{r}, N_{\theta}}$ will converge to the exact solution $U(r, \theta)$ exponentially fast as $N_{r}, N_{\theta} \rightarrow+\infty$ provided that all $F(r, \theta)$, $\xi(\theta)$, and $\eta(\theta)$ are analytic in $\Omega$, and (ii) our numerical scheme is stable for large $N_{r}$ and capable of providing accurate results for moderate to large wave numbers.

To summarize, we have presented a complete analysis for the spectral-Galerkin method to the Helmholtz equation in exterior domains. We first studied asymptotic behaviors of the Hankel functions which play essential roles for our error analysis. Using these asymptotic estimates, we then derived a priori estimates with explicit dependence on the wave number for both the continuous and the discrete problems. Finally, we performed an error analysis and derived error bounds with explicit dependence on the wave number. To the authors' best knowledge, our error estimates seem to be the first of their kind, i.e., with explicit dependence on the wave number for a numerical method on bounded obstacle scattering via the DtN map. A particular advantage of this approach, verified by our error estimates and numerical results, is that we can choose the artificial boundary very close to the scatterer while still maintaining the spectral accuracy.

Appendix A. The proof of (2.34). We first prove (2.34a). It is clear that, by $(2.33 \mathrm{~b}), \operatorname{Im}\left(\mathcal{T}_{m, \kappa}\right)>0$. On the other hand, since $\operatorname{Im}\left(\mathcal{T}_{m, \kappa}\right)$ is a strictly increasing 
(resp., decreasing) function of $\kappa$ (resp., $m$ ), we have

$$
\operatorname{Im}\left(\mathcal{T}_{m, \kappa}\right) \leq \operatorname{Im}\left(\mathcal{T}_{1, \kappa}\right)<\operatorname{Im}\left(\mathcal{T}_{1, \infty}\right)=1
$$

due to the asymptotic formula $\left|H_{1}^{(1)}(\kappa)\right|^{2} \sim \frac{2}{\pi \kappa}$ for $\kappa \gg 1$ (see Formula 9.2.3 of [1]).

We now turn to the proof of $(2.34 \mathrm{~b})$. Recall that the modified Bessel function of the second kind of order $\nu$ is defined by

$$
\mathcal{K}_{\nu}(z)=\int_{0}^{\infty} e^{-z \cosh t} \cosh (\nu t) d t
$$

In particular, we have

$$
\mathcal{K}_{0}(z)=\int_{0}^{\infty} e^{-z \cosh t} d t, \quad \mathcal{K}_{1}(z)=-\mathcal{K}_{0}^{\prime}(z)
$$

By Formula (4) on p. 445 of [31],

$$
\begin{gathered}
J_{m}^{2}(\kappa)+Y_{m}^{2}(\kappa)=\frac{8}{\pi^{2}} \int_{0}^{\infty} \mathcal{K}_{0}(2 \kappa \sinh t) \cosh (2 m t) d t \\
{\left[J_{m} J_{m+1}+Y_{m} Y_{m+1}\right](\kappa)=\frac{8}{\pi^{2}} \int_{0}^{\infty} \mathcal{K}_{1}(2 \kappa \sinh t) \sinh ((2 m+1) t) d t}
\end{gathered}
$$

Using the identity $\mathcal{K}_{1}(z)=-\mathcal{K}_{0}^{\prime}(z)$ and integration by parts leads to

$$
\begin{aligned}
{\left[J_{m} J_{m+1}+\right.} & \left.Y_{m} Y_{m+1}\right](\kappa)=-\frac{8}{\pi^{2}} \int_{0}^{\infty} \frac{\sinh ((2 m+1) t)}{2 \kappa(\sinh t)^{\prime}} d\left(\mathcal{K}_{0}(2 \kappa \sinh t)\right) \\
= & -\left.\frac{4}{\kappa \pi^{2}} \frac{\sinh ((2 m+1) t)}{\cosh t} \mathcal{K}_{0}(2 \kappa \sinh t)\right|_{0} ^{\infty} \\
& +\frac{4}{\kappa \pi^{2}} \int_{0}^{\infty} \mathcal{K}_{0}(2 \kappa \sinh t)\left(\frac{\sinh ((2 m+1) t)}{\cosh t}\right)^{\prime} d t \\
= & \frac{4}{\kappa \pi^{2}} \int_{0}^{\infty} \mathcal{K}_{0}(2 \kappa \sinh t) \cosh (2 m t) W_{m}(t) d t
\end{aligned}
$$

where

$$
W_{m}(t)=\frac{1}{\cosh (2 m t)}\left(\frac{\sinh ((2 m+1) t)}{\cosh t}\right)^{\prime}
$$

Note that in the last step we used the asymptotic formula (see Formula 9.7. 2 of [1])

$$
\mathcal{K}_{0}(2 \kappa \sinh t) \sim \sqrt{\frac{\pi}{2 \kappa \sinh t}} e^{-2 \kappa \sinh t} \sim e^{-\kappa e^{t}-t / 2}, \quad t \gg 1,
$$

to claim that

$$
\frac{\sinh ((2 m+1) t)}{\cosh t} \mathcal{K}_{0}(2 \kappa \sinh t) \rightarrow 0 \quad \text { as } t \rightarrow \infty
$$

Using the identities of the hyperbolic functions, $W_{m}(t)$ can be written as

$$
W_{m}(t)=2 m(1+(\tanh t) \tanh (2 m t))+\operatorname{sech}^{2} t, \quad 0 \leq t<\infty .
$$

Copyright (C) by SIAM. Unauthorized reproduction of this article is prohibited. 
We now seek the maximum and minimum values of $W_{m}(t)$. Taking the derivative of $W_{m}(t)$ yields

$$
\begin{aligned}
W_{m}^{\prime}(t) & =2 m\left(\left(\operatorname{sech}^{2} t\right) \tanh (2 m t)+2 m(\tanh t) \operatorname{sech}^{2}(2 m t)\right)-2(\tanh t) \operatorname{sech}^{2} t \\
& =2(m \tanh (2 m t)-\tanh t) \operatorname{sech}^{2} t+4 m^{2}(\tanh t) \operatorname{sech}^{2}(2 m t) .
\end{aligned}
$$

It is obvious that

$$
m \tanh (2 m t)-\tanh t>0 \quad \forall t>0, \forall m \geq 1 .
$$

Hence, $W_{m}(t)$ is an increasing function of $t$, and consequently,

$$
2 m+1=W_{m}(0) \leq W_{m}(t) \leq W_{m}(\infty)=4 m \quad \forall t \geq 0, \forall m \geq 1 .
$$

Therefore, for $m \geq 1$,

$$
\frac{2 m+1}{2 \kappa} \leq \frac{J_{m}(\kappa) J_{m+1}(\kappa)+Y_{m}(\kappa) Y_{m+1}(\kappa)}{J_{m}^{2}(\kappa)+Y_{m}^{2}(\kappa)} \leq \frac{2 m}{\kappa},
$$

which, together with (2.33a), yields the bounds

$$
-\frac{m}{\kappa} \leq \operatorname{Re}\left(\mathcal{T}_{m, \kappa}\right) \leq-\frac{1}{2 \kappa} \quad \text { for } \quad m \geq 1
$$

Finally, for $m=0$, (A.5) implies that $0<W_{0}(t) \leq 1$. Accordingly, we find that

$$
-\frac{1}{2 \kappa} \leq \operatorname{Re}\left(\mathcal{T}_{0, \kappa}\right)<0
$$

It remains to prove $(2.34 \mathrm{c})$.

Let us first show that $\operatorname{Im}\left(\mathcal{T}_{0, \kappa}\right)$ is a strictly decreasing function of $\kappa$. By $(2.33 \mathrm{~b})$, it suffices to show that

$$
f(x):=x\left|H_{0}^{(1)}(x)\right|^{2}=x\left(J_{0}^{2}(x)+Y_{0}^{2}(x)\right), \quad x>0,
$$

is a strictly increasing function of $x$. Indeed, by (A.3a),

$$
f(x)=\frac{8}{\pi^{2}} \int_{0}^{\infty} x \mathcal{K}_{0}(2 x \sinh t) d t
$$

Differentiating it gives

$$
f^{\prime}(x)=\frac{8}{\pi^{2}} \int_{0}^{\infty}\left\{\mathcal{K}_{0}(2 x \sinh t)+2 x \sinh t \mathcal{K}_{0}^{\prime}(2 x \sinh t)\right\} d t
$$

Integrating the second term by parts leads to

$$
f^{\prime}(x)=\left.\frac{8}{\pi^{2}} \mathcal{K}_{0}(2 x \sinh t) \tanh t\right|_{0} ^{\infty}+\frac{8}{\pi^{2}} \int_{0}^{\infty} \mathcal{K}_{0}(2 x \sinh t) \tanh ^{2} t d t .
$$

Notice that the first term is zero due to the decay property of $\mathcal{K}_{0}$ (cf. (A.4)). Therefore, we have

$$
f^{\prime}(x)>0 \quad \forall x>0 .
$$

Finally, (2.34c) follows immediately from $(2.33 \mathrm{~b})$ and the facts that $\operatorname{Im}\left(\mathcal{T}_{0, \kappa}\right)$ is a strictly decreasing function of $\kappa$ and $\kappa\left|H_{0}^{(1)}(\kappa)\right|^{2} \rightarrow \frac{2}{\pi}$ as $\kappa \rightarrow \infty$.

This ends the proof of (2.34). 


\section{REFERENCES}

[1] M. Abramowitz and I. A. Stegun, eds., Handbook of Mathematical Functions with Formulas, Graphs, and Mathematical Tables, New York, 1984. Reprint of the 1972 edition, Selected Government Publications.

[2] M. Ainsworth, Discrete dispersion relation for hp-version finite element approximation at high wave number, SIAM J. Numer. Anal., 42 (2004), pp. 553-575.

[3] M. Ainsworth, Dispersive and dissipative behaviour of high order discontinuous Galerkin finite element methods, J. Comput. Phys., 198 (2004), pp. 106-130.

[4] I. M. BABUŠKA AND S. A. SAUTER, Is the pollution effect of the FEM avoidable for the Helmholtz equation considering high wave numbers?, SIAM J. Numer. Anal., 34 (1997), pp. 23922423.

[5] O. P. Bruno and F. Reitich, Numerical solution of diffraction problems: A method of variation of boundaries, J. Opt. Soc. Amer. A, 10 (1993), pp. 1168-1175.

[6] O. P. BRuno And F. Reitich, Numerical solution of diffraction problems: A method of variation of boundaries. II. Finitely conducting gratings, Padé approximants, and singularities, J. Opt. Soc. Amer. A, 10 (1993), pp. 2307-2316.

[7] C. Canuto, M. Y. Hussaini, A. Quarteroni, and T. A. Zang, Spectral Methods in Fluid Dynamics, Springer-Verlag, Berlin, 1987.

[8] S. N. Chandler-Wilde and S. Langdon, A Galerkin boundary element method for high frequency scattering by convex polygons, SIAM J. Numer. Anal., 45 (2007), pp. 610-640.

[9] S. N. Chandler-Wilde And P. Monk, Existence, uniqueness, and variational methods for scattering by unbounded rough surfaces, SIAM J. Math. Anal., 37 (2005), pp. 598-618.

[10] P. Cummings And X. Feng, Sharp regularity coefficient estimates for complex-valued acoustic and elastic Helmholtz equations, Math. Models Methods Appl. Sci., 16 (2006), pp. 139-160.

[11] L. Demkowicz And F. Ihlenburg, Analysis of a coupled finite-infinite element method for exterior Helmholtz problems, Numer. Math., 88 (2001), pp. 43-73.

[12] J. Douglas, J. E. Santos, D. Sheen, and L. S. Bennethum, Frequency domain treatment of one-dimensional scalar waves, Math. Models Methods Appl. Sci., 3 (1993), pp. 171-194.

[13] M. J. Grote and J. B. Keller, On nonreflecting boundary conditions, J. Comput. Phys., 122 (1995), pp. 231-243.

[14] B. GUO AND L. WANG, Jacobi approximations in non-uniformly Jacobi-weighted Sobolev spaces, J. Approx. Theory, 128 (2004), pp. 1-41.

[15] I. Harari and T. J. R. Hughes, Analysis of continuous formulations underlying the computation of time-harmonic acoustics in exterior domains, Comput. Methods Appl. Mech. Engrg., 97 (1992), pp. 103-124.

[16] F. Ihlenburg, Finite Element Analysis of Acoustic Scattering, Appl. Math. Sci. 132, SpringerVerlag, New York, 1998.

[17] F. IhlenBurg AND I. BABUŠKa, Finite element solution of the Helmholtz equation with high wave number, part I: The h-version of FEM, Comput. Math. Appl., 30 (1995), pp. 9-37.

[18] F. Ihlenburg AND I. BABUŠKA, Finite element solution of the Helmholtz equation with high wave number, part II: The h-p version of the FEM, SIAM J. Numer. Anal., 34 (1997), pp. $315-358$.

[19] S. Langdon and S. N. Chandler-Wilde, A wavenumber independent boundary element method for an acoustic scattering problem, SIAM J. Numer. Anal., 43 (2006), pp. 24502477.

[20] J.-L. Lions and E. Magenes, Non-homogeneous Boundary Value Problems and Applications, Vol. I, Springer-Verlag, New York, 1972 (translated from the French by P. Kenneth, Die Grundlehren der mathematischen Wissenschaften, Band 181).

[21] J. M. Melenk, On Generalized Finite Element Methods, Ph.D. thesis, The University of Maryland, College Park, MD, 1995.

[22] P. Monk, Finite Element Methods for Maxwell's Equations, Numer. Math. Sci. Comput., Oxford University Press, New York, 2003.

[23] J.-C. NÉDÉLEC, Acoustic and Electromagnetic Equations, Appl. Math. Sci. vol. 144, SpringerVerlag, New York, 2001.

[24] D. P. Nicholls and J. Shen, A stable, high-order method for two-dimensional boundedobstacle scattering, SIAM J. Sci. Comput., 28 (2006), pp. 1398-1419.

[25] D. P. Nicholls and F. Reitich, Stability of high-order perturbative methods for the computation of Dirichlet-Neumann operators, J. Comput. Phys., 170 (2001), pp. 276-298.

[26] D. P. Nicholls And F. Reitich, Shape deformations in rough surface scattering: Improved algorithms, J. Opt. Soc. Amer. A, 21 (2004), pp. 606-621.

Copyright (C) by SIAM. Unauthorized reproduction of this article is prohibited. 
[27] L. RaYleigh, On the dynamical theory of gratings, Proc. R. Soc. Lond. Ser. A, 79 (1907), pp. 399-416.

[28] J. SHEn, Efficient spectral-Galerkin method I. Direct solvers of second- and fourth-order equations using Legendre polynomials, SIAM J. Sci. Comput., 15 (1994), pp. 1489-1505.

[29] J. Shen, Efficient spectral-Galerkin methods III: Polar and cylindrical geometries, SIAM J. Sci. Comput., 18 (1997), pp. 1583-1604.

[30] J. Shen And L.-L. WAng, Spectral approximation of the Helmholtz equation with high wave numbers, SIAM J. Numer. Anal., 43 (2005), pp. 623-644.

[31] G. N. Watson, A Treatise on the Theory of Bessel Functions, 2nd ed., Cambridge University Press, Cambridge, UK, 1966.

Copyright (c) by SIAM. Unauthorized reproduction of this article is prohibited. 